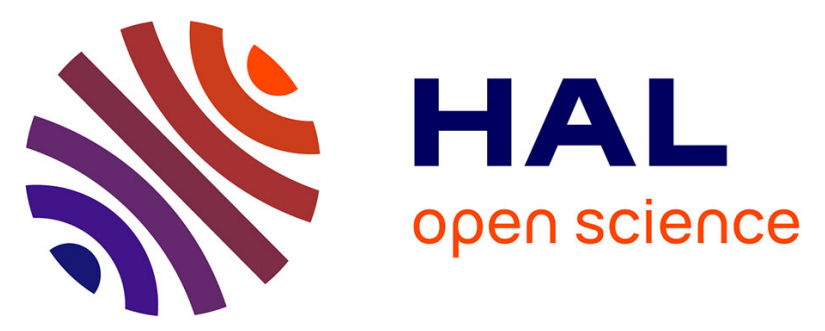

\title{
Rotating magnetic field effect on convection and its stability in a horizontal cylinder subjected to a longitudinal temperature gradient
}

\author{
D.V. Lyubimov, A.V. Burnysheva, Hamda Ben Hadid, T.P. Lyubimova, \\ Daniel Henry
}

\section{To cite this version:}

D.V. Lyubimov, A.V. Burnysheva, Hamda Ben Hadid, T.P. Lyubimova, Daniel Henry. Rotating magnetic field effect on convection and its stability in a horizontal cylinder subjected to a longitudinal temperature gradient. Journal of Fluid Mechanics, 2010, 664, pp.108-137. 10.1017/S0022112010003678 . hal-00566050

\section{HAL Id: hal-00566050 https://hal.science/hal-00566050}

Submitted on 8 Jun 2012

HAL is a multi-disciplinary open access archive for the deposit and dissemination of scientific research documents, whether they are published or not. The documents may come from teaching and research institutions in France or abroad, or from public or private research centers.
L'archive ouverte pluridisciplinaire HAL, est destinée au dépôt et à la diffusion de documents scientifiques de niveau recherche, publiés ou non, émanant des établissements d'enseignement et de recherche français ou étrangers, des laboratoires publics ou privés. 


\title{
Rotating magnetic field effect on convection and its stability in a horizontal cylinder subjected to a longitudinal temperature gradient
}

\author{
D. V. LYUBIMOV ${ }^{1}$, A. V. BURNYSHEVA ${ }^{2}$, H. BEN HADID ${ }^{3}$, \\ T. P. LYUBIMOVA ${ }^{2}$ AND D. HENRY ${ }^{3} \dagger$ \\ ${ }^{1}$ Perm State University, 15 Bukirev Street, 614990 Perm, Russia \\ ${ }^{2}$ Institute of Continuous Media Mechanics UB RAS, 1 Koroleva Street, \\ 614013 Perm, Russia \\ ${ }^{3}$ Laboratoire de Mécanique des Fluides et d'Acoustique, Université de Lyon, \\ Ecole Centrale de Lyon, Université Lyon 1, INSA de Lyon, \\ UMR-CNRS 5509, 43 Bd du 11 Novembre 1918, \\ 69622 Villeurbanne CEDEX, France
}

(Received 22 March 2010; revised 6 July 2010; accepted 6 July 2010; first published online 15 October 2010)

A rotating magnetic field (RMF) is used in crystal growth applications during the solidification process in order to improve the crystal quality. Its influence on the convective flows in molten metals and on their stability is studied here in the case of a horizontal infinite cylindrical channel subjected to a longitudinal temperature gradient. The steady convective flows, which correspond to the usual longitudinal counterflow structure, with four vortices in the cross-section for non-zero Prandtl number, $P r$, are modified by the RMF (parametrized by the magnetic Taylor number $\left.T a_{m}\right)$. For zero Prandtl number, the flow in the cross-section corresponds to circular streamlines and the longitudinal flow structure is moved in the direction of the magnetic field rotation, with a decrease in its intensity and an asymptotic variation as $1 / T a_{m}$. For non-zero Prandtl numbers, depending on the respective values of $T a_{m}$ on one side and Prandtl and Grashof numbers on the other side, different structures ranging from the circular streamlines with transport by rotation of the longitudinal velocity and the temperature field, to the more usual counterflow structure almost insensitive to the RMF with four cross-section vortices, can be obtained. The decrease in the flow intensity with increasing $T a_{m}$ is also delayed for non-zero $\mathrm{Pr}$, but the same asymptotic limit is eventually reached. The stability analysis of these convective flows for $T a_{m}=0$ shows a steep increase of the thresholds around $P r=P r_{t, 0} \approx 3 \times 10^{-4}$, corresponding to the transition between the usual counterflow shear mode and a new sidewall shear mode. This transition is still present with an RMF, but it occurs for smaller $\mathrm{Pr}$ values as $T a_{m}$ is increased. Strong stabilizing effects of the rotating magnetic field are found for $P r<P r_{t, 0}$, particularly for $P r=0$ where an exponential increase of the threshold with $T a_{m}$ is found. For $\operatorname{Pr}>P r_{t, 0}$ (i.e. in the domain where the sidewall instability is dominant), in contrast, the stabilization by the RMF is weak.

Key words: convection in cavities, instability, magnetohydrodynamics 


\section{Introduction}

During the growth of a single crystal from a melt, oscillatory flows are generated in the melt, which lead to fluctuations in the convective heat transfer from the melt to the crystal and to fluctuations in the rate of solidification. The dopant segregation in the crystal is affected and spatial oscillations of the dopant concentration in the crystal, called striations, can appear (Müller \& Wiehelm 1964). The control of the melt motions is then necessary to improve the quality of the crystal. Both static magnetic fields and rotating magnetic fields (RMFs) can be used for the flow control in the melt, and the benefits which can be obtained in terms of crystal quality are described by Utech \& Flemings (1966) and Dold \& Benz (1999), respectively. The two fields, however, act on the melt flow in different ways: the static magnetic field has rather a braking effect whereas the RMF induces a rotating motion in the melt. In this paper, we will consider an RMF and see how it can modify the flow properties in a differentially heated cylindrical cavity typical of the horizontal Bridgman growth technique.

Convection in enclosures with differentially heated vertical boundaries is typical of many engineering systems involving heat transfers such as crystal growth, solar energy collection, the welding process, nuclear reactors, and energy efficient design of buildings. The stability of such convection in low-Prandtl-number fluids typical of crystal growth has been widely investigated in the literature. The first study concerned the buoyant flows generated by a horizontal temperature gradient in a fluid layer confined between two horizontal walls of infinite extent (Hart 1972; Laure \& Roux 1987; Kuo \& Korpela 1988) and different instabilities were found depending on the Prandtl number Pr. Two-dimensional finite length enclosures heated from the side (GAMM workshop, Roux 1990) were then considered. The stability of the flows in such situations was investigated by different authors as Winters (1988), Skeldon, Riley \& Cliffe (1996), and Gelfgat, Bar-Yoseph \& Yarin (1999). These studies clearly illustrated that for two-dimensional enclosures, there are different perturbation modes which become the more dangerous when the longitudinal aspect ratio (length/height) is varied. A few numerical simulations concern three-dimensional parallelepipedic enclosures (Henry \& Buffat 1998; Wakitani 2001; Henry \& Ben Hadid 2007). These studies (see particularly Henry \& Ben Hadid 2007) pointed out that the critical Grashof number for the occurrence of time-dependent flows and the type of perturbations involved are strongly dependent on the Prandtl number and the cavity aspect ratios (length/height and width/height). The experimental studies of the time-dependent flows in such enclosures are few. After the pioneering study of Hurle, Jakeman \& Johnson (1974), which reports measurements of temperature oscillations in molten gallium in a cavity with moderate aspect ratios (typically $2.5 \times 1.1 \times 1$ for length $\times$ width $\times$ height), we can mention the study of Kamotani \& Sahraoui (1990) in mercury-filled cavities, which points out the influence of the aspect ratios on the onset of oscillations, and the more recent studies of Braunsfurth \& Mullin (1996) and Hof et al. (2004) in gallium-filled cavities, which rather focus their studies on the influence of the Prandtl number. These studies also point out the difficulties in controlling the thermal properties of the boundaries.

Compared to all these results obtained for rectangular cavities, the similar convection problem in horizontal circular cylindrical cavities has received less attention. Concerning the structure of the steady flow, we can mention the analytical approach of Bejan \& Tien (1978), who give an asymptotic solution for the velocity and temperature distributions in the middle portion of a long horizontal pipe with a conducting wall, and the numerical approach of Bontoux et al. (1986), who point out a transition between a core-driven regime and a boundary-layer-driven regime when 
the temperature difference between the two ends of the cylinder is increased. The three-dimensional structure of the flow is also studied in the experiment of Davoust et al. (1999) in a mercury-filled cell submitted to a vertical magnetic field. Large values of the Grashof number induce a boundary-layer-driven regime which transits to a core-driven regime when the magnetic field intensity is increased. Concerning the transition to oscillatory flows in low-Prandtl-number fluids, interesting results have been obtained numerically by Vaux, Ben Hadid \& Henry (2006). The aspect ratio $A(A=$ length/diameter $)$ of the cylinder $(1.5 \leqslant A \leqslant 10)$ is shown to strongly affect the onset of oscillatory flows: the corresponding critical Grashof number strongly decreases when $A$ is increased from $A=1.5$ to 3 , then strongly increases until $A=8$ and eventually decreases more slowly until $A=10$.

The RMF used in most crystal-growth processes is a spatially uniform, transverse magnetic field which rotates at a constant angular velocity, $\omega$, around the centreline of the cylindrical cavity containing the melt. The electromagnetic body force which is produced consists of a steady axisymmetric azimuthal force, and a periodic three-dimensional force which is negligible in crystal-growth applications (Martin Witkowski, Walker \& Marty 1999). Experimental observations of Dold \& Benz (1997) indicate that the RMF can lead to modifications of fluid flow and heat transport in vertical Bridgman configurations. Values of field strength of a few milliteslas are sufficient to damp temperature fluctuations in the melt and to decrease striation amplitudes in the crystal, whereas more than 100 milliteslas are required in the case of a static magnetic field (Dold \& Benz 1995). When the intensity of the RMF is further increased, oscillatory flows can be triggered. Different studies have determined the stability of the rotating flow induced by the RMF. The first study used time-stepping codes in a two-dimensional axisymmetric approximation (Barz et al. 1997; Kaiser \& Benz 1998; Mössner \& Gerbeth 1999). They found an increase of the critical magnetic Taylor number $T a_{m}$ (based on the radius) when the aspect ratio (height/radius) is decreased. A discrepancy exists between the results, which shows the difficulties in computing such situations. Grants \& Gerbeth (2001) directly solved the stability problem in axisymmetric conditions and found linear thresholds associated to perturbations which are not Taylor-Görtler vortices. Small finiteamplitude perturbations in the form of Taylor-Görtler vortices, however, can give rise to instability in the linearly stable regime. This can explain the discrepancy between the previous results. Grants \& Gerbeth (2002) then accounted for three-dimensional perturbations and found that the most dangerous perturbations at the linear stage are not axisymmetric but correspond to modes with azimuthal wavenumbers $m=1$, 2 or 3, depending on the aspect ratio. Three-dimensional simulations by Ben Hadid, Vaux \& Kaddeche (2001) mention the appearance of steady Taylor-Görtler vortices at values of the magnetic Taylor number which can be correlated with the aspect ratio of the cavity. Oscillatory instabilities appear for stronger magnetic Taylor numbers: two types of instabilities with very different frequencies are found for increasing magnetic Taylor numbers, but unexpected restabilizations are found in intermediate $T a_{m}$ ranges.

The influence of the RMF on buoyant flows is considered numerically in a simple configuration by Mössner \& Gerbeth (1999): a stabilizing influence is obtained which is interpreted as the result of partly counterbalancing flows. The main studies concern the Rayleigh-Bénard configuration. Volz \& Mazuruk (1999) first presented a linear stability analysis for an infinitely long cylinder. They demonstrated that the RMF increases the critical Rayleigh number for asymmetric flow modes but does not affect the onset of instability for axisymmetric modes, and that the instability first 


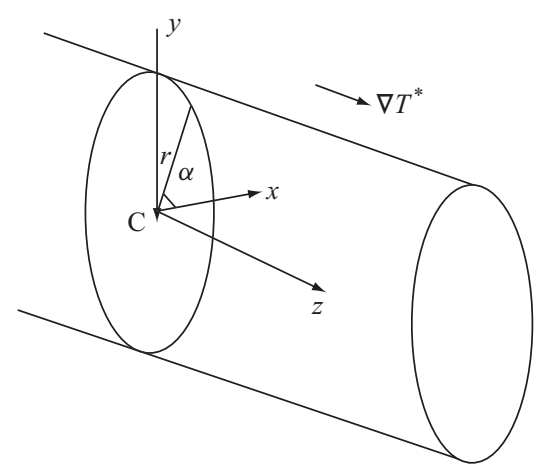

Figure 1. Configuration studied.

develops in the form of a single asymmetric meridional roll rotating around the axis of the cylinder. Volz \& Mazuruk (2001) then presented an experimental study corresponding to liquid gallium contained in a cylinder heated from below with aspect ratio (height/diameter) equal to 1 , which is a main contribution in this field. For small magnetic Taylor numbers the critical Rayleigh number at which instabilities are triggered is strongly increased with respect to its value without an RMF and the observed flow is consistent with a single roll rotating around the cylinder axis. For large magnetic Taylor numbers, in contrast, the stationary flow domain is bounded by a critical value of the magnetic Taylor number, which is independent of the Rayleigh number.

In this paper, we want to study the influence of RMFs on the convective flows in a differentially heated horizontal cylindrical cavity typical of the horizontal Bridgman crystal growth technique. As fully three-dimensional numerical calculations to simulate such convective flows in long cylinders (Vaux et al. 2006) or to take into account the flows generated by the RMF (Ben Hadid et al. 2001) are still difficult to perform with sufficient accuracy, we choose to derive a model which considers the three components of the velocity and the temperature in the cross-section of an infinitelength cylindrical channel. Both steady-state calculations and stability analyses with respect to fully three-dimensional perturbations are performed. Such a method was previously used in the case of a rectangular cross-section channel and for an applied static magnetic field (Lyubimova et al. 2009; Lyubimov et al. 2009). The base state will be first studied in detail, both analytically and numerically, to see how the convective flow structure is changed when the RMF is applied. A linear stability analysis is then performed to determine how the base state is destabilized by small three-dimensional perturbations periodical in the direction of the channel axis, and to investigate the dependence of the instability thresholds and the perturbation structures on the Prandtl and magnetic Taylor numbers.

\section{Formulation of the problem, governing equations and boundary conditions}

We consider the convective flows of an electrically conducting fluid with density $\rho$, kinematic viscosity $v$ and electrical conductivity $\sigma$ in a horizontal cylinder with circular cross-section of radius $R$ (figure 1). A uniform horizontal temperature gradient $(\nabla T)^{*}$ is imposed at the lateral boundary, and the system is subjected to a uniform magnetic field of induction $B$ orthogonal to the cylinder axis and rotating around this axis with a constant angular frequency $\omega$. 
The models developed to describe flows of conducting liquids in an RMF have been presented in many previous studies (for example in Barz et al. 1997; Kaiser \& Benz 1998; Martin Witkowski et al. 1999). For crystal growth applications, it has been shown that the magnetic field variations in the melt are negligible and that the flow does not influence the driving magnetic body force. The first condition is principally fulfilled because the skin depth (penetration length of the RMF) is much larger than the radius of the cylinder, which can be expressed by $\left(\mu_{p} \sigma \omega\right)^{-1 / 2} \gg R$ or $\mu_{p} \sigma \omega R^{2} \ll 1$, where $\mu_{p}$ is the magnetic permeability of the melt. The influence of the velocity in the melt on the magnetic field also has to be small, which corresponds to low magnetic Reynolds number $R e_{m}=\mu_{p} \sigma V_{c} R\left(V_{c}\right.$ is a characteristic velocity in the melt), a hypothesis really well-verified for melts in laboratory conditions (Moreau 1990). The second condition implies that the angular frequency is much larger than any typical angular velocity in the melt, i.e. $\omega \gg V_{c} / R$. This condition can be expressed in terms of the field characteristics as $H a^{2} R e_{\omega}^{-1} \ll 1$, where $H a=B R(\sigma /(\rho v))^{1 / 2}$ is the Hartmann number and $R e_{\omega}=\omega R^{2} / v$ is a Reynolds number associated with the magnetic field rotation.

With these conditions, the time-averaged magnetic body force (which strongly dominates the oscillating force part; see Martin Witkowski et al. 1999) has only an azimuthal component. In the case of an infinitely long cylinder, this force depends linearly on the radius $r^{*}$ and can be written as $\boldsymbol{F}=0.5 \sigma \omega B^{2} r^{*} \boldsymbol{e}_{\alpha}$, where $\boldsymbol{e}_{\alpha}$ is the unit vector in the azimuthal direction.

The governing equations of the problem are the Navier-Stokes equations taking into account the magnetic body force, coupled to the energy equation. Using $R$, $R^{2} / \nu, v / R, \rho(\nu / R)^{2}$ and $(\nabla T)^{*} R$ as scales for length, time, velocity, pressure and temperature, respectively, these equations in their dimensionless form are

$$
\begin{gathered}
\frac{\partial \boldsymbol{V}}{\partial t}+(\boldsymbol{V} \cdot \nabla) \boldsymbol{V}=-\nabla P+\nabla^{2} \boldsymbol{V}+G r T \boldsymbol{\gamma}+T a_{m} r \boldsymbol{e}_{\alpha}, \\
\frac{\partial T}{\partial t}+\boldsymbol{V} \cdot \nabla T=\frac{1}{P r} \nabla^{2} T, \\
\nabla \cdot \boldsymbol{V}=0,
\end{gathered}
$$

where the dimensionless variables are the velocity $\boldsymbol{V}=(\boldsymbol{U}, W)(\boldsymbol{U}$ is the velocity in the cross-section, $W$ is the longitudinal velocity along the $z$-coordinate), the temperature $T$ and the pressure $P$. The non-dimensional parameters are the Grashof number, $G r=g \beta(\nabla T)^{*} R^{4} / \nu^{2}$, the Prandtl number, $P r=\nu / \kappa$ and the magnetic Taylor number, $T a_{m}=\omega \sigma B^{2} R^{4} /\left(2 \rho v^{2}\right)$. In these expressions, $g$ is the acceleration due to gravity, $\beta$ is the thermal expansion coefficient and $\kappa$ is the thermal diffusivity. $\gamma$ is the unit vector directed vertically upwards along the $y$-direction. The associated boundary conditions applied on $r=1$ are no-slip conditions and a fixed temperature distribution (purely conducting walls), i.e. $\boldsymbol{V}=0$ and $T=z$ (conductive profile). An additional condition to solve the problem comes from mass conservation in the cylinder cross-section $S$, $\int_{S} W \mathrm{~d} S=0$.

\section{Basic flow}

As in Lyubimova et al. (2009), we look for the basic flow solution in which the velocity and the temperature deviation from the conductive state do not depend on $z$ :

$$
\boldsymbol{U}=\boldsymbol{U}(r, \alpha), \quad W=W(r, \alpha), \quad T=z+\Theta(r, \alpha),
$$


where $r, \alpha$ and $z$ are the cylindrical coordinates. Then the pressure $P$ is a linear function of $z$ (Lyubimova et al. 2009):

$$
P=\operatorname{Gr} r z \sin \alpha+K z+\Pi(r, \alpha),
$$

where $K$ is a constant. (Note that we have assumed that the origin of the angular coordinate $\alpha$ is the horizontal $x$-axis.) Accounting for (3.1) and (3.2), we can write (2.1)-(2.3) in the form

$$
\begin{gathered}
\frac{\partial \boldsymbol{U}}{\partial t}+(\boldsymbol{U} \cdot \nabla) \boldsymbol{U}=-\nabla \Pi+\nabla^{2} \boldsymbol{U}+G r \Theta \boldsymbol{\gamma}+T a_{m} r \boldsymbol{e}_{\alpha}, \\
\frac{\partial W}{\partial t}+(\boldsymbol{U} \cdot \nabla W)=-G r r \sin \alpha-K+\nabla^{2} W, \\
\frac{\partial \Theta}{\partial t}+(\boldsymbol{U} \cdot \nabla \Theta)+W=\frac{1}{P r} \nabla^{2} \Theta, \\
\nabla \cdot \boldsymbol{U}=0 .
\end{gathered}
$$

Note that in these equations the differentiations are restricted to the cross-section plane. The constant $K$ is determined from mass conservation in the channel crosssection $S, \int_{S} W \mathrm{~d} S=0$, which gives

$$
K=\frac{1}{S} \int_{S} \nabla^{2} W \mathrm{~d} S=\frac{1}{S} \oint_{\Gamma} \frac{\partial W}{\partial n} \mathrm{~d} \Gamma,
$$

where $\Gamma$ is the boundary of the cylinder cross-section $S$ and $n$ is the normal to the cylinder surface.

In $\S 3.3$, we will see that both in the absence and in the presence of a magnetic field, the basic flow solution exhibits a symmetry $S_{c}$ with respect to the centre point of the channel cross-section. For the basic flow, mass conservation is then automatically satisfied and $K=0$.

To calculate the basic flow in the general case of non-zero Prandtl number, it is useful to introduce the stream function $\psi$ and the vorticity $\Omega$ (related to the flow in the cross-section, $\boldsymbol{U}=(U, V))$ which are such that

$$
U=\frac{1}{r} \frac{\partial \psi}{\partial \alpha}, \quad V=-\frac{\partial \psi}{\partial r}, \quad \Omega=(\nabla \times \boldsymbol{U})_{z}=-\nabla_{s}^{2} \psi .
$$

The governing equations for the steady basic flow written in terms of stream function and vorticity are

$$
\begin{gathered}
\boldsymbol{U} \cdot \nabla_{s} \Omega=\nabla_{s}^{2} \Omega+G r \frac{\partial \Theta}{\partial x}+2 T a_{m}, \\
\boldsymbol{U} \cdot \nabla_{s} W=\nabla_{s}^{2} W-G r r \sin \alpha, \\
\operatorname{Pr} \boldsymbol{U} \cdot \nabla_{s} \Theta=\nabla_{s}^{2} \Theta-\operatorname{Pr} W \\
\nabla_{s}^{2} \psi=-\Omega,
\end{gathered}
$$

with boundary conditions $\psi=\partial \psi / \partial r=\Theta=W=0$ at $r=1$. In these equations, we use the differential operators $\nabla_{s}=\boldsymbol{e}_{r}(\partial / \partial r)+\boldsymbol{e}_{\alpha}(1 / r)(\partial / \partial \alpha)$, where $\boldsymbol{e}_{r}$ is the unit vector in the radial direction, and $\nabla_{s}^{2}=\left(\partial^{2} / \partial r^{2}\right)+(1 / r)(\partial / \partial r)+\left(1 / r^{2}\right)\left(\partial^{2} / \partial \alpha^{2}\right)$.

The basic flow equations (3.9)-(3.12) in cylindrical coordinates are then discretized using a finite difference method with second-order spatial derivatives and solved by the Newton method. For the calculation at the singular point $r=0$, the equations are written in Cartesian coordinates and the derivatives along $x$ and $y$ are estimated from the values of the fields taken at $r=\delta r$ for $\alpha=0$ and $\pi$, and $\alpha=\pi / 2$ and $3 \pi / 2$, 

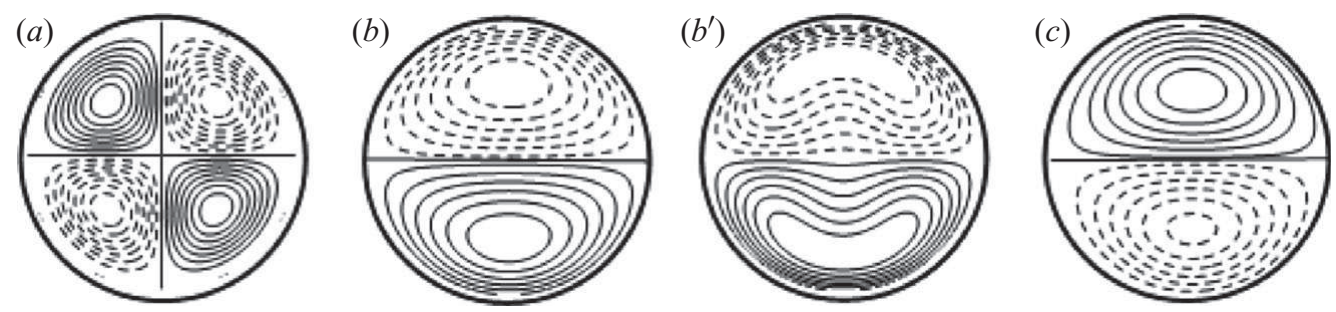

FIGURE 2. Streamlines of the transverse flow $(a)$, isolines of the longitudinal velocity $\left(b, b^{\prime}\right)$ and isolines of the temperature field $(c)$ for the basic solution at the critical threshold without magnetic field $\left(T a_{m}=0\right)$. $P r=0.00001\left(G r_{c}=5307\right)$ for $(a-c), \operatorname{Pr}=0.001\left(G r_{c}=37466\right)$ for $\left(b^{\prime}\right)$.

respectively $(r=\delta r$ represents the circle of mesh points close to $r=0)$. The zero state or the solution obtained at a smaller value of the Grashof number is used as an initial guess for the Newton solver. The linear system (with band structure) obtained at each Newton step is solved by the elimination method. Typical grids of $40 \times 160$ points (in radial and azimuthal directions, respectively) have been used to calculate the basic solutions. The accuracy of the numerical solutions obtained for non-zero $\operatorname{Pr}$ has been checked by comparison with analytical solutions. We can give two examples: the curve of $W_{\max } / G r$ versus $T a_{m}$ numerically calculated for $P r=0.0001$ is found to be very close to that obtained analytically for $P r=0$ in figure 3; the numerical solutions obtained for $T a_{m}=40000$ in figure 6 verify the analytical predictions derived for large $T a_{m}$.

\subsection{Basic flow in the absence of a magnetic field}

In the case of horizontal cylinders of rectangular cross-section (Lyubimova et al. 2009), it has been shown that for zero Prandtl number, i.e. when the temperature field is 'frozen' to its conductive profile, the problem has a solution which corresponds to a plane-parallel flow $(W \neq 0, \boldsymbol{U}=0)$ proportional to $G r$. For any non-zero values of the Prandtl number, in contrast, the plane-parallel flow does not exist: all three components of the flow velocity differ from zero and four vortices develop in the cylinder cross-section. All these observations are also valid in the case of a cylinder of circular cross-section.

Examples of the basic steady flow characteristics are shown in figure 2 for $\operatorname{Pr}=$ 0.00001 and 0.001. The plots are given for values of $G r$ corresponding to the onset of instabilities. In these plots, the solid lines indicate positive values and the dashed lines indicate negative values. The global flow consists of a large circulation, where the fluid moves in the direction of the imposed temperature gradient in the lower part of the cavity and in the opposite direction in the upper part. The interface between the two counterflows is horizontal (figure $2 b$ ). A closer view on the isotherms in the cross-section shows that the convective circulation drives hot fluid from the warmer area in the upper part of the cross-section, whereas it drives cold fluid from the colder area in the lower part. This creates a stable vertical stratification (figure $2 c$ ). The four vortices which appear in the cross-section (figure $2 a$ ) are a consequence of the temperature variations in the cross-section, between the core and the sidewalls. In these vortices, the fluid circulates from the centre point of the cross-section to the periphery in the vertical direction and back from the periphery to the centre point in the horizontal direction. The temperature variations in the cross-section, as well as the intensity of the vortices, grow proportionally to Pr. For large enough values of 
$\operatorname{Pr}$ (and subsequent values of $G r$ ), the vortices can even lead to modifications of the longitudinal flow structure (figure $2 b^{\prime}$ ).

\subsection{Rotating magnetic field effect on the basic flow}

\subsubsection{Zero Prandtl number}

We first study the effect of the RMF on the basic flow in the case of zero Prandtl number. Without an RMF, there is no flow in the cross-section for $\mathrm{Pr}=0$ $(\boldsymbol{U}=0)$. With an RMF, the flow in the cross-section, solution of (3.3) with $\Theta=0$, is axisymmetric and given by

$$
\boldsymbol{U}=\frac{1}{8} T a_{m}\left(1-r^{2}\right) r \boldsymbol{e}_{\alpha} .
$$

This flow is the same as that generated by the RMF in the absence of heating and corresponds to circular streamlines in the cross-section. Substituting this solution into (3.5), we obtain the following equation for the longitudinal velocity $W$ :

$$
\frac{1}{8} T a_{m}\left(1-r^{2}\right) \frac{\partial W}{\partial \alpha}=\nabla_{s}^{2} W-G r r \sin \alpha .
$$

We look for the solution of (3.14) in the form

$$
W(r, \alpha)=\operatorname{Gr} \operatorname{Im}\left(\mathscr{W}(r) \mathrm{e}^{\mathrm{i} \alpha}\right),
$$

where $\operatorname{Im}$ denotes the imaginary part, and thus obtain an equation for $\mathscr{W}(r)$,

$$
\mathscr{W}^{\prime \prime}+\frac{1}{r} \mathscr{W}^{\prime}-\frac{1}{r^{2}} \mathscr{W}+\frac{\mathrm{i}}{8} T a_{m}\left(r^{2}-1\right) \mathscr{W}=r,
$$

with the boundary condition

$$
\mathscr{W}=0 \quad \text { at } r=1 .
$$

The solutions of this equation are expressed through the Whittaker functions and integrals of these functions.

Small values of the magnetic Taylor number. For small values of $T a_{m}$, the solution of (3.16) and (3.17) can be represented in the form of series with respect to $T a_{m}$,

$$
\mathscr{W}=\mathscr{W}_{0}+T a_{m} \mathscr{W}_{1}+\cdots
$$

where

$$
\begin{gathered}
\mathscr{W}_{0}=\frac{1}{8} r\left(r^{2}-1\right), \\
\mathscr{W}_{1}=\frac{\mathrm{i}}{3072} r\left(1-r^{2}\right)\left(r^{4}-3 r^{2}+3\right) .
\end{gathered}
$$

At zero order, we then have

$$
W_{0}(r, \alpha)=\frac{G r}{8} r\left(r^{2}-1\right) \sin \alpha .
$$

This solution describes the convective flow at $P r=0$ in the absence of a magnetic field. It corresponds to parallel counterflows separated by a horizontal interface at $y=0$, similar to what is shown in figure $2(b)$ for $\operatorname{Pr}=0.00001$. The effect of the magnetic field at first order is described by

$$
W_{1}(r, \alpha)=\frac{G r T a_{m}}{3072} r\left(1-r^{2}\right)\left(r^{4}-3 r^{2}+3\right) \cos \alpha .
$$

The fact that $\mathscr{W}_{1}$ is purely imaginary (or the factor $\cos \alpha$ in $W_{1}$ ) indicates that, in the presence of a magnetic field, the interface between the counterflows will move 


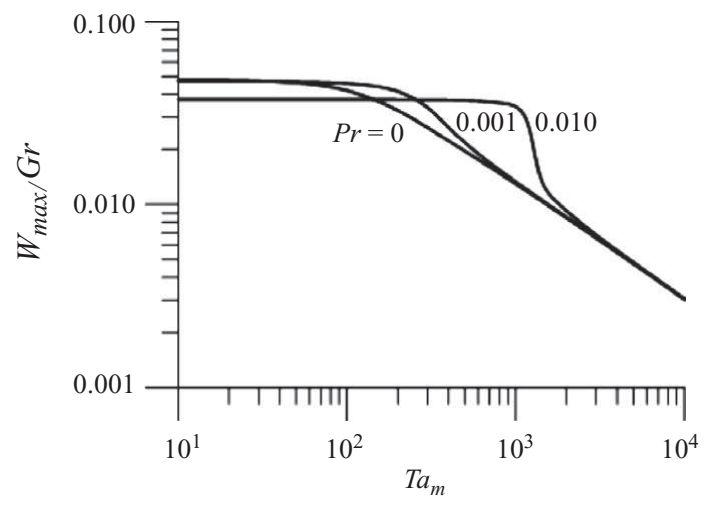

FIGURE 3. Maximal longitudinal velocity $\left(W_{\max } / G r\right)$ as a function of the magnetic Taylor number $T a_{m}$ for three values of the Prandtl number $(P r=0,0.001$ and 0.01$)$. For non-zero $P r, W_{\max } / G r$ which still depends on $G r$ has been plotted for $G r=10000$.

from its horizontal position at $T a_{m}=0$ and become inclined. This inclination of the interface occurs in the direction of the magnetic field rotation and is due to the drag induced by the circular flow $\boldsymbol{U}$ (3.13). Finally, note that the ratio of $W_{1}$ and $W_{0}$ amplitudes is given by $\left|W_{1}\right| /\left|W_{0}\right| \approx T a_{m} / 384$.

Limit case of large values of the magnetic Taylor number. In the limit case of large $T a_{m}$, the solution of (3.16) and (3.17) is of the boundary-layer type. For the bulk flow, retaining only the leading terms in (3.16), we obtain

$$
\mathscr{W}=\frac{8 \mathrm{i}}{T a_{m}} \frac{r}{1-r^{2}} .
$$

At first non-vanishing order, the bulk flow is then expressed by

$$
W(r, \alpha)=\frac{8 G r}{T a_{m}} \frac{r}{1-r^{2}} \cos \alpha .
$$

The factor $\cos \alpha$ indicates that, in this approximation of large $T a_{m}$, the interface between the counterflows is vertical in the bulk. Moreover, the flow intensity decreases with the increase of $T a_{m}$, i.e. the RMF suppresses the convective flow.

Finite values of the magnetic Taylor number. For finite values of $T a_{m}$, the solution of (3.16) is expressed through the Whittaker functions. A numerical estimate of the longitudinal velocity $W$ in the cross-section is thus obtained.

In figure 3, we can see the dependence of the maximal $W$ velocity on the magnetic Taylor number for $\mathrm{Pr}=0$. As $W$ is proportional to $\mathrm{Gr}$ for $\mathrm{Pr}=0$, we chose to plot $W_{\max } / G r$ which is independent of $G r$. (Note that the plot is given in a log-log scale.) We see that the asymptotic behaviour at large $T a_{m}$, which corresponds to $W_{\max } \sim\left(1 / T a_{m}\right)$, is already observed for $T a_{m}>200$. The isolines of the longitudinal velocity $W$ are then presented for different values of the magnetic Taylor number in figure 4. As expected from the previous approximations, we see that with the increase of $T a_{m}$ the flow structure is moved by the magnetic field rotation and the flow becomes concentrated near the circular boundary, whereas in the weak flow remaining in the bulk, the interface between the two counterflows becomes progressively vertical. Again note that at $\operatorname{Pr}=0$ the structure of the flow only depends on the 


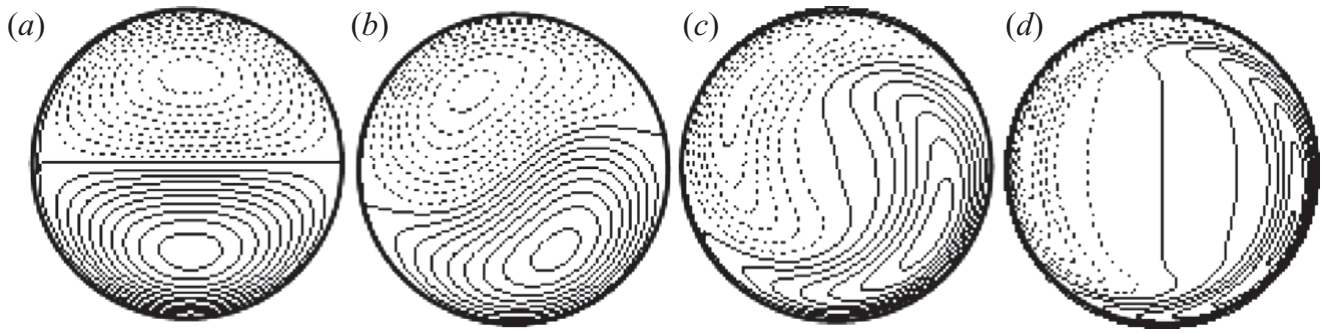

FIGURE 4. Isolines of the longitudinal velocity $W$ for different values of the magnetic Taylor number $T a_{m}$ in the case of zero Prandtl number: (a) $T a_{m}=0$, (b) $T a_{m}=100,(c) T a_{m}=1000$ and $(d) T a_{m}=10000$.

magnetic Taylor number, whereas its intensity also linearly depends on the Grashof number.

\subsubsection{Small values of the Prandtl number}

In the case of small $P r$, the temperature field (at first order in $P r$ ) is determined by the equation

$$
\nabla_{s}^{2} \Theta=\operatorname{Pr} W .
$$

Accounting for (3.15), we seek a solution for $\Theta$ in the form

$$
\Theta(r, \alpha)=\operatorname{Pr} \operatorname{Gr} \operatorname{Im}\left(\vartheta(r) \mathrm{e}^{\mathrm{i} \alpha}\right) .
$$

We obtain an equation for $\vartheta(r)$,

$$
\vartheta^{\prime \prime}+\frac{1}{r} \vartheta^{\prime}-\frac{1}{r^{2}} \vartheta=\mathscr{W},
$$

with boundary conditions

$$
\vartheta(0)=0, \quad \vartheta(1)=0 .
$$

The solution of equations (3.27) and (3.28) can be expressed as

$$
\vartheta(r)=\frac{1}{2} \int_{0}^{1}\left(r^{2} \mathscr{W}(r x)-r \mathscr{W}(x)\right)\left(1-x^{2}\right) \mathrm{d} x,
$$

which, in particular, gives

$$
\vartheta^{\prime}(0)=-\frac{1}{2} \int_{0}^{1} \mathscr{W}(x)\left(1-x^{2}\right) \mathrm{d} x .
$$

Small values of the magnetic Taylor number. At small $T a_{m}$, the rotation effect will be small and we can assume that the drag induced through $\boldsymbol{U}$ will not perturb $W, \Theta$ and $\Omega$. Equation (3.10) for $W$ is then reduced to

$$
\nabla_{s}^{2} W=G r r \sin \alpha,
$$

which leads to the solution $W_{0}$ (see (3.21)) already encountered at zero order in $T a_{m}$ for $P r=0$.

The temperature deviation $\Theta$ is the solution of (3.11) which is reduced to (3.25). The solution $\Theta_{1}$ is obtained by substituting $\mathscr{W}_{0}$ given by (3.19) in (3.29) and using (3.26). We get

$$
\Theta_{1}=\frac{\operatorname{Pr} G r}{192} r\left(r^{2}-1\right)\left(r^{2}-2\right) \sin \alpha,
$$



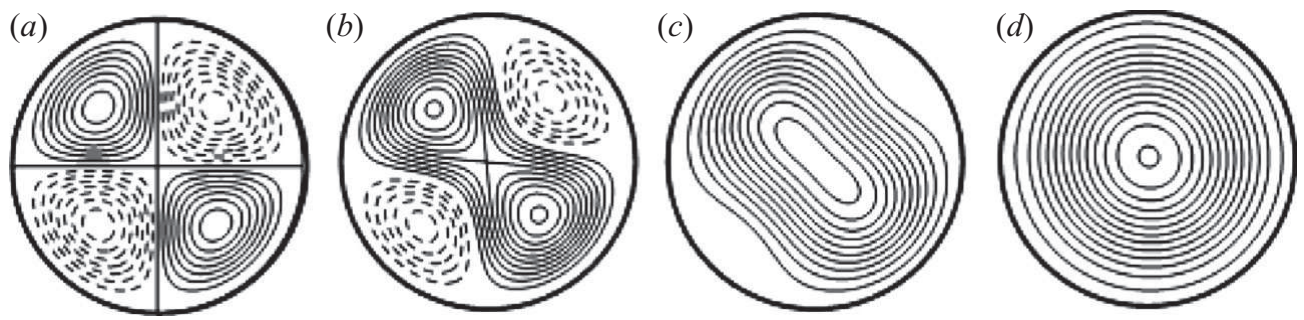

FIGURE 5. Streamlines of the transverse flow for different values of the parameter $a=$ $\left(11520 T a_{m}\right) /\left(\operatorname{Pr} G r^{2}\right)$ in the small $\operatorname{Pr}$ approximation and for small values of $\operatorname{Ta}_{m}:(a) a=0$, (b) $a=1,(c) a=5$ and (d) $a=100$.

and using (3.9) and (3.12), we obtain the stream function $\psi_{1}$ :

$$
\psi_{1}=\frac{\operatorname{PrGr}^{2}}{368640} r^{2}\left(2 r^{2}-11\right)\left(r^{2}-1\right)^{2} \sin 2 \alpha+\frac{1}{32} T a_{m}\left(r^{2}-1\right)^{2},
$$

which is the summation of two contributions, the first one coming from buoyancy and the second one coming from the RMF. The ratio of these contributions (magnetic/buoyancy) is characterized by the parameter $a=\left(11520 T a_{m}\right) /\left(\operatorname{Pr} r^{2}\right)$. The isolines of the stream function for different values of $a$ are plotted in figure 5. We see the regular change of the flow structure from the four vortices induced by buoyancy to the circular motion created by the RMF when $a$ is increased. Note that the rotation effect due to the magnetic field is already dominant for $a=5$. As an example, for $P r=0.01$ and $G r=10000$, this corresponds to $T a_{m} \approx 434$.

Limit case of large values of the magnetic Taylor number. In the limit case of large $T a_{m}$, but if we still assume that (3.25) is valid (small $\mathrm{Pr}$ approximation), by substituting (3.23) into (3.29) and using (3.26), we obtain

$$
\Theta(r, \alpha)=\frac{2 \operatorname{Pr} G r}{T a_{m}} \frac{1-r^{2}}{r} \ln \left(1-r^{2}\right) \cos \alpha .
$$

In this small $\operatorname{Pr}$ approximation, the temperature deviation $\Theta$ in the bulk only follows the modifications induced by the RMF on the longitudinal velocity $W$. The interface between the hot and cold zones in the bulk is vertical, as is the interface between the counterflows.

\subsubsection{Finite values of the Prandtl number}

For finite values of $P r$, we will first see the limit case of large $T a_{m}$ values before considering the more general situation with finite values of $T a_{m}$.

Limit case of large values of the magnetic Taylor number. For large values of $T a_{m}$ and finite values of $P r$, the leading terms for the transversal velocities $\boldsymbol{U}$ and the longitudinal velocity $W$ in the bulk are given by (3.13) and (3.24), respectively. The drag induced by $\boldsymbol{U}$ on $\Theta$ is important in this case and the energy equation (3.11) can be written as

$$
\boldsymbol{U} \cdot \nabla_{s} \Theta=-W
$$

from which we can easily find the expression of $\Theta$ in the bulk:

$$
\Theta(r, \alpha)=-\frac{64 G r}{T a_{m}^{2}} \frac{\sin \alpha}{\left(1-r^{2}\right)^{2}} .
$$



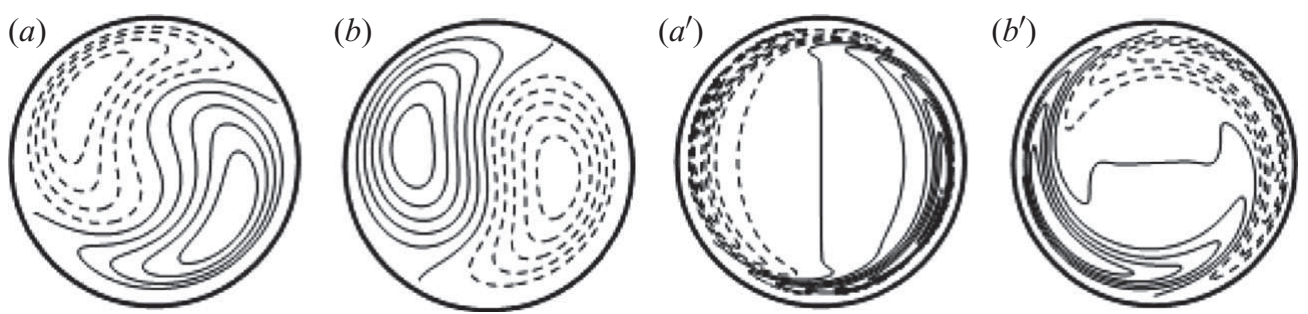

FIGURE 6. Isolines of the longitudinal velocity $\left(a, a^{\prime}\right)$ and isolines of the temperature field $\left(b, b^{\prime}\right)$ for the basic solution at $\operatorname{Pr}=0.1$ and $G r=100$ for $T a_{m}=500(a, b)$ and $T a_{m}=40000$ $\left(a^{\prime}, b^{\prime}\right)$.

We see that in this case the interface between the hot and cold zones is horizontal. This result differs from the result found for large $T a_{m}$ in the small $\mathrm{Pr}$ approximation (see (3.34)) which indicated a vertical interface. In fact, the relevant parameter for the different approximations of the temperature field at large $T a_{m}$ is the product $\operatorname{Pr} T a_{m}$ appearing in the term $\operatorname{Pr} \boldsymbol{U} \cdot \nabla_{s} \Theta$ of the energy equation when $\boldsymbol{U}$ is given by (3.13). The asymptotic expression (3.34) associated to the energy equation (3.25) is obtained for small values of this product, whereas the asymptotic expression (3.36) associated to the energy equation (3.35) is obtained for large values of this product. In the first case, the temperature distribution is governed by diffusive heat transfers, but it follows the modifications induced by the RMF on $W$. This gives a rotation of $\pi / 2$ of the interface in the bulk. In the second case, in contrast, the temperature distribution is governed by convective heat transfers (the parameter $\mathrm{Pr}$ related to thermal dffusivity is not present in the expression (3.36)), except in the domain near the boundary where thermal boundary layers will develop. The temperature field in the bulk will still follow the modifications induced on $W$, but will also be transported by the rotating flow $\boldsymbol{U}$. This now gives a rotation of $\pi$ of the interface in the bulk, as indicated by the change of sign of $\Theta$ between the expression (3.32) for small $T a_{m}$ and the expression (3.36). This behaviour is illustrated in figure 6 by the results obtained numerically for $P r=0.1$ and $G r=100$. We see the rotation of the longitudinal velocity and the temperature field with respect to their structure at $T a_{m}=0$ (figures $2 b$ and $2 c$ ) as $T a_{m}$ is increased. For $T a_{m}=500$, both fields are quite similarly affected by the RMF with an interface which is close to vertical in the bulk. We can guess that, at this stage, the temperature field mainly follows the deformations of the longitudinal velocity. For $T a_{m}=40000$, in contrast, the transport by rotation of the temperature field is stronger, as it has still progressed, whereas the transport of the longitudinal velocity has not much evolved, except that we are now in a boundary layer regime with variations of the fields concentrated along the lateral wall. At this stage, both asymptotic behaviours for the interfaces in the bulk (vertical for the longitudinal velocity and horizontal for the temperature field) can be observed.

Finite values of the magnetic Taylor number. For finite values of both $\mathrm{Pr}$ and $\mathrm{Ta} \mathrm{m}_{m}$, (3.9)-(3.12) are solved numerically. In the following, we want to study the influence of the RMF on the basic flow in the more general case of non-zero Prandtl number.

We first analyse the effect of the magnetic Taylor number on the intensity of the basic flow. The variation of $W_{\max } / G r$ with $T a_{m}$ is plotted for $\mathrm{Pr}=0.001$ and 0.01 in figure 3. Note that these plots have been obtained for a given value of $\mathrm{Gr}$ $(G r=10000)$, because for $\operatorname{Pr} \neq 0, W_{\max } / G r$ still depends on $G r$. We first see that for this value of $G r, W_{\max } / G r$ decreases as $\operatorname{Pr}$ is increased in the case without a magnetic 
field. The braking effect due to the RMF is also delayed as $\mathrm{Pr}$ is increased. This is particularly visible for $P r=0.01$, where values of $T a_{m}$ larger than 1000 are necessary to initiate the braking of the flow. The further evolutions of $W_{\max } / G r$ with $T a_{m}$ show a decrease, which is steeper in the situations with larger $P r$ where this decrease was delayed. The curves eventually merge with the asymptotic part of the curve obtained at $P r=0$, which indicates an eventual braking of the flow as $\left(1 / T a_{m}\right)$. It is thus shown that, for large enough values of $T a_{m}$, the thermal effects associated with non-zero Prandtl numbers become negligible.

We now consider the variation of the basic flow structure with the characteristic parameters. We study the effect of $\mathrm{Pr}$ for fixed values of $T a_{m}$ and $\mathrm{Gr}\left(T a_{m}=100\right.$, $G r=10000)$. The structure of the flow at $P r=0$ corresponds to circular streamlines in the cross-section (given by (3.13)), as those shown in figure $5(f)$, and to the longitudinal velocity field shown in figure 4 for $T a_{m}=100$. The increase of $\mathrm{Pr}$ diminishes the influence of the RMF. Indeed, for $P r=0.001$, both a decrease of the rotation in the cross-section and a decrease of the inclination of the longitudinal flow are obtained, and the temperature field follows the inclination of the longitudinal velocity field with less distortion, and for $P r=0.01$, the structure of the basic flow looks quite similar to that obtained without a magnetic field and shown in figure $2\left(a, b^{\prime}, c\right)$, with only symmetry properties which are not perfectly verified. In fact, an increase (decrease) of $\mathrm{Pr}$ and $\mathrm{Gr}$ acts on the flow structures in the same way as a decrease (increase) of $T a_{m}$. This is what could be expected from the small $\mathrm{Pr}$ approximation (\$3.2.2) where the parameters appear as $\operatorname{Pr}^{2} r^{2} / T a_{m}$ (in $\psi$ for small $T a_{m}$ ) or $\mathrm{PrGr} / \mathrm{Ta} a_{m}$ (in $\Theta$ for large $T a_{m}$ ).

\subsection{Symmetries of the problem}

The geometry is considered as infinite in the $z$-direction of the cylinder axis and the basic flow does not depend on $z$. The problem is thus invariant under translations $T_{z_{0}}$ by length $z_{0}$ along the $z$-axis. The group generated by the translations is the group $E(1)$. In the case without an RMF, the problem admits two supplementary symmetries: a symmetry of rotation of $\pi$ about any horizontal axis parallel to the $x$-direction, for example the $x$-axis, which we denote by $R_{\pi}$, and a reflection symmetry $S_{l}$ with respect to the longitudinal vertical plane (the plane along the $z$-axis, at $x=0$, denoted as the $V_{l}$ plane). With an RMF, $R_{\pi}$ and $S_{l}$ are no more valid, but the problem admits another supplementary symmetry with respect to any point belonging to the $z$-axis, for example the point $C$ at the intersection with the $x$-axis, which we denote as $S_{c}$. Note that $S_{c}=R_{\pi} \circ S_{l}$, so that $S_{c}$ is also verified in the case without a magnetic field. $R_{\pi}, S_{l}$ and $S_{c}$ are defined, respectively, as

$$
\begin{aligned}
R_{\pi}:(x, y, z, t) & \rightarrow(x,-y,-z, t), \quad(U, V, W, T) \rightarrow(U,-V,-W, T), \\
S_{l}:(x, y, z, t) & \rightarrow(-x, y, z, t), \quad(U, V, W, T) \rightarrow(U,-V, W, T), \\
S_{c}:(x, y, z, t) & \rightarrow(-x,-y,-z, t), \quad(U, V, W, T) \rightarrow(U, V,-W, T) .
\end{aligned}
$$

(Remember that $U$ and $V$ are the radial and azimuthal velocity components.) The symmetries $R_{\pi}$ and $S_{c}$ are equivalent to two and three combined reflections, respectively. Each symmetry $R_{\pi}, S_{l}$ and $S_{c}$ defines a $Z_{2}$ group.

In the case without a magnetic field, the symmetry group, generated by $T_{z_{0}}, R_{\pi}$ and $S_{l}$, is isomorphic to $E(1) \times Z_{2} \times Z_{2}$, whereas in the case with an RMF, it is generated by $T_{z_{0}}$ and $S_{c}$ and is isomorphic to $E(1) \times Z_{2}$. All these symmetries may be broken at the instability thresholds where bifurcations to new flow states will occur. 


\section{Stability of the basic steady convective flow}

\subsection{Linear stability analysis}

The stability of the basic flows described in $\S 3$ is now investigated by a linear analysis. These basic flows are steady and uniform in the direction of the channel axis (z-direction). The general solution of the three-dimensional problem can then be written as the superposition of the basic flow and normal-mode perturbations periodic in the $z$-direction:

$$
(\boldsymbol{V}, P, T)=\left(\boldsymbol{V}_{0}, P_{0}, T_{0}\right)+(\boldsymbol{v}, p, \theta) \mathrm{e}^{(\omega t+\mathrm{i} k z)},
$$

where $k$ is the wavenumber in the $z$-direction and $\omega=\omega_{r}+i \omega_{i}$ is a complex growth rate (temporal growth rate $\omega_{r}$ and oscillation frequency $\omega_{i}$ ). After substitution of (4.1) into the governing system (3.3)-(3.6) and linearization with respect to the perturbation quantities, we obtain the following system of linear equations:

$$
\begin{aligned}
& \omega u+U_{0} \frac{\partial u}{\partial r}+\frac{V_{0}}{r} \frac{\partial u}{\partial \alpha}+\mathrm{i} k W_{0} u-\frac{V_{0} v}{r}+u \frac{\partial U_{0}}{\partial r}+\frac{v}{r} \frac{\partial U_{0}}{\partial \alpha}-\frac{v V_{0}}{r} \\
& =-\frac{\partial p}{\partial r}+\left(\nabla_{s}^{2}-k^{2}\right) u-\frac{u}{r^{2}}-\frac{2}{r^{2}} \frac{\partial v}{\partial \alpha}+G r \theta \sin \alpha, \\
& \omega v+U_{0} \frac{\partial v}{\partial r}+\frac{V_{0}}{r} \frac{\partial v}{\partial \alpha}+\mathrm{i} k W_{0} v+\frac{V_{0} u}{r}+u \frac{\partial V_{0}}{\partial r}+\frac{v}{r} \frac{\partial V_{0}}{\partial \alpha}+\frac{v U_{0}}{r} \\
& =-\frac{1}{r} \frac{\partial p}{\partial \alpha}+\left(\nabla_{s}^{2}-k^{2}\right) v-\frac{v}{r^{2}}+\frac{2}{r^{2}} \frac{\partial u}{\partial \alpha}+G r \theta \cos \alpha, \\
& \omega w+U_{0} \frac{\partial w}{\partial r}+\frac{V_{0}}{r} \frac{\partial w}{\partial \alpha}+\mathrm{i} k W_{0} w+u \frac{\partial W_{0}}{\partial r}+\frac{v}{r} \frac{\partial W_{0}}{\partial \alpha}=-\mathrm{i} k p+\left(\nabla_{s}^{2}-k^{2}\right) w, \\
& \omega \theta+U_{0} \frac{\partial \theta}{\partial r}+\frac{V_{0}}{r} \frac{\partial \theta}{\partial \alpha}+\mathrm{i} k W_{0} \theta+u \frac{\partial T_{0}}{\partial r}+\frac{v}{r} \frac{\partial T_{0}}{\partial \alpha}+w=\frac{1}{P r}\left(\nabla_{s}^{2}-k^{2}\right) \theta, \\
& \frac{\partial u}{\partial r}+\frac{u}{r}+\frac{1}{r} \frac{\partial v}{\partial \alpha}+\frac{\partial w}{\partial z}=0,
\end{aligned}
$$

with boundary conditions

$$
u=v=w=\theta=0 \quad \text { at } \quad r=1 .
$$

Here $U_{0}, V_{0}, W_{0}$ and $u, v, w$ are the velocity components (along radial, azimuthal and longitudinal directions) of the basic flow and the perturbations, respectively. $T_{0}$ is the temperature of the basic state, and $\theta$ and $p$ are the temperature and pressure perturbations, respectively.

Using the same discretization as for the basic state, we obtain a generalized eigenvalue problem $\boldsymbol{A} \boldsymbol{X}=\omega \boldsymbol{B} \boldsymbol{X}$, where $\boldsymbol{A}$ is a complex matrix, $\boldsymbol{B}$ is a real symmetric matrix and $\boldsymbol{X}$ is the vector of the discretized perturbations. To solve this problem and thus obtain the most critical eigenvalues and the corresponding eigenvectors (fields of velocity, temperature and pressure perturbations), a Newton-Raphson method is used, which is initiated by the result of a linearized time evolution (see Lyubimova et al. 2009 and Lyubimov, Lyubimova \& Morozov 2001 for details). The neutral perturbations corresponding to a marginal Grashof number are then obtained from the condition $\omega_{r}=0$ (zero growth rate). Minimization over the wavenumber $k$ yields the critical values $k_{c}$ and $G r_{c}$.

\subsection{Energy analysis}

In order to get some physical insight into the stability results, we performed energy analyses. For that, by multiplying the linear stability equations by the complex 
conjugate of the velocity perturbation, $\boldsymbol{v}^{*}$, and after integration on the cross-section $S$ and some simplifications, an equation giving the rate of change of the fluctuating kinetic energy can be derived. From this equation, for any instabilities at their critical thresholds $\left(\omega_{r}=0\right)$, normalized kinetic energy budgets can be obtained, which we can write as:

$$
E_{s}^{\prime}+E_{b}^{\prime}=1,
$$

where

$$
E_{s}^{\prime}=\frac{-\operatorname{Re}\left(\sum_{i=1}^{3} \sum_{j=1}^{2} \int_{S} v_{j} \frac{\partial V_{0 i}}{\partial x_{j}} v_{i}^{*} \mathrm{~d} S\right)}{\left|E_{d}\right|}
$$

are the productions of fluctuating kinetic energy by shear of the basic flow and

$$
E_{b}^{\prime}=\frac{\operatorname{Re}\left(\int_{S} G r \theta v_{2}^{*} \mathrm{~d} S\right)}{\left|E_{d}\right|}
$$

is the production of fluctuating kinetic energy by buoyancy, all these terms being normalized by the viscous dissipation of fluctuating kinetic energy,

$$
E_{d}=\operatorname{Re}\left(\sum_{i=1}^{3} \sum_{j=1}^{3} \int_{S} \frac{\partial^{2} v_{i}}{\partial x_{j}^{2}} v_{i}^{*} \mathrm{~d} S\right) .
$$

In these expressions, the basic flow and the velocity perturbations are expressed in Cartesian coordinates $\left(x, y, z\right.$ or $x_{i}$ for $i=1$ to 3$)$ and they are denoted as $V_{0 i}$ and $v_{i}$, respectively (note that $V_{03}=W_{0}$ and $v_{3}=w$ ). $R e$ denotes the real part.

We will see that the two important shear terms in our situation are those related to the basic longitudinal velocity. The first one, which is dominant without an RMF (see Lyubimova et al. 2009), is the term related to the vertical variation of $W_{0}, E_{s y}^{\prime}=-\operatorname{Re}\left(\int_{S} v_{2}\left(\partial W_{0} / \partial y\right) w^{*} \mathrm{~d} S\right) /\left|E_{d}\right|$. The second one is the term related to the transverse variation of $W_{0}, E_{s x}^{\prime}=-\operatorname{Re}\left(\int_{S} v_{1}\left(\partial W_{0} / \partial x\right) w^{*} \mathrm{~d} S\right) /\left|E_{d}\right|$. These terms are obtained by integration of spatial fields, $\mathscr{E}_{s y}^{\prime}=\operatorname{Re}\left(\left(-\partial W_{0} / \partial y\right) v_{2} w^{*}\right) /\left|E_{d}\right|$ and $\mathscr{E}_{s x}^{\prime}=\operatorname{Re}\left(\left(-\partial W_{0} / \partial x\right) v_{1} w^{*}\right) /\left|E_{d}\right|$, respectively. These fields can also be expressed as the product of two terms, respectively, related to the basic flow and the fluctuating flow. These terms are $M_{y}=\left(-\partial W_{0} / \partial y\right)$ and $F_{y}=\operatorname{Re}\left(v_{2} w^{*}\right) /\left|E_{d}\right|$ for $\mathscr{E}_{s y}^{\prime}$, and $M_{x}=\left(-\partial W_{0} /\right.$ $\partial x)$ and $F_{x}=\operatorname{Re}\left(v_{1} w^{*}\right) /\left|E_{d}\right|$ for $\mathscr{E}_{s x}^{\prime}$. We can then write $E_{s y}^{\prime}=\int_{S} \mathscr{E}_{s y}^{\prime} \mathrm{d} S=\int_{S} M_{y} F_{y} \mathrm{~d} S$ and $E_{s x}^{\prime}=\int_{S} \mathscr{E}_{s x}^{\prime} \mathrm{d} S=\int_{S} M_{x} F_{x} \mathrm{~d} S$.

\section{Stability results}

\subsection{Convective flow stability in the absence of a rotating magnetic field}

The linear stability of the convective flow in the cylinder with respect to threedimensional perturbations is first studied without a magnetic field for a wide range of Prandtl number values. First, note that for $P r=0$ the critical Grashof number corresponding to the instability threshold is $G r_{c}=5263$ and the critical wavenumber is $k_{c}=0.34$. In this case, the temperature field is frozen (no temperature perturbations) and the instability is steady and of hydrodynamic type. The structure of the critical perturbation is shown in figure 7. 
(a)

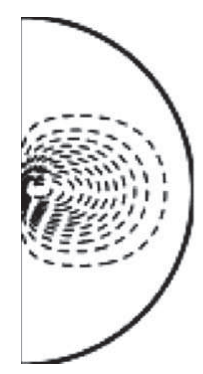

(b)

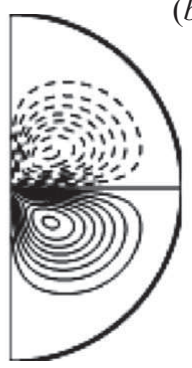

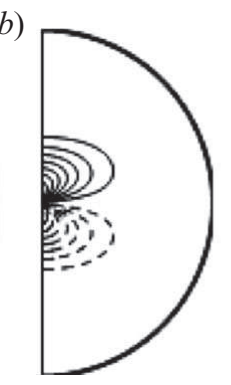

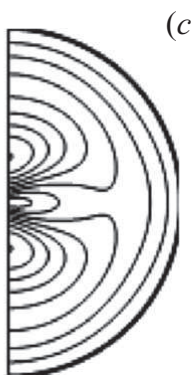

(c)

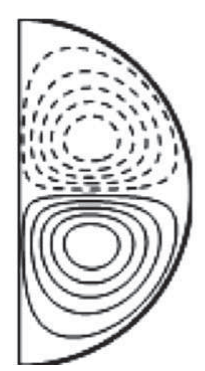

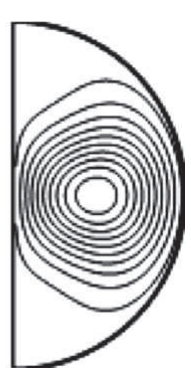

FIGURE 7. Isolines for the real and imaginary parts of the radial $(a)$, azimuthal $(b)$ and longitudinal $(c)$ velocity perturbations at the critical threshold $\left(G r_{c}=5263, k_{c}=0.34\right)$ for $P r=0$ and without magnetic field $\left(T a_{m}=0\right)$. Because of the symmetry properties, only half of the cross-section is shown.

As explained in $\S 3.3$, the symmetry group of our problem in the absence of an RMF is generated by $T_{z_{0}}, R_{\pi}$ and $S_{l}$. Each eigenmode with finite wavelength $\left(k_{c} \neq 0\right)$ breaks the translation invariance and is thus associated with a bifurcation which produces an infinite number of equivalent steady states parametrized by $z_{0}$. Each eigenmode possesses horizontal axes of symmetry (separated by half a wavelength, $\lambda / 2=\pi / k)$ and axes of antisymmetry at mid-length between the symmetry axes; one of the symmetry axes can be chosen to be the $x$-axis (at $z=0$ ) so as to make the eigenmode symmetric under $R_{\pi}$. If the discretized eigenvector is denoted as $X_{r}+\mathrm{i} X_{i}$, the perturbation will be given by $\operatorname{Re}\left(X \mathrm{e}^{\mathrm{i} k z}\right)=X_{r} \cos (k z)-X_{i} \sin (k z)$. According to the symmetries just mentioned, $X_{r}$, which is the perturbation in the cross-section at $z=0$, will appear symmetric with respect to the horizontal axis at $y=0$, whereas $X_{i}$, which is the perturbation in the cross-section at $z=\pi /(2 k)=\lambda / 4$, will appear antisymmetric. In intermediate cross-sections, the perturbation will not present any symmetry with respect to the horizontal axis at $y=0$. This is illustrated in figure 7 where the real and imaginary parts of the eigenvectors for the velocities $u, v$ and $w$ are given. We see that $v_{r}$ and $w_{r}$ appear as odd in $y$ and $u_{r}$ as even (this expresses the symmetry), whereas $v_{i}$ and $w_{i}$ appear as even in $y$ and $u_{i}$ as odd (this expresses the antisymmetry).

The eigenvectors plotted in figure 7 also indicate that the symmetry $S_{l}$ with respect to the vertical plane $V_{l}$ at $x=0$ is broken at the critical threshold $\left(u_{r}, u_{i}, w_{r}, w_{i}\right.$ are odd in $x$ and $v_{r}, v_{i}$ are even). This result contrasts with those obtained for a rectangular cross-section (Lyubimova et al. 2009), which showed that this left-right symmetry was kept at the critical threshold for cavities with a transverse aspect ratio ranging from 1 to 5 . Finally, the eigenmode has points of symmetry (separated by half a wavelength) on the $z$-axis and points of antisymmetry in between. As $S_{c}=R_{\pi} \circ S_{l}$ and $S_{l}$ is broken, the point at $z=0$ appears as a point of antisymmetry and the point at $z=\lambda / 4$ as a point of symmetry.

Without an RMF $\left(T a_{m}=0\right)$, the stability curve showing the dependence of the critical Grashof number $\mathrm{Gr}_{c}$ (for the more dangerous steady hydrodynamic mode) on the Prandtl number $\mathrm{Pr}$ and the curve giving the corresponding variation of the critical wavenumber $k_{c}$ are shown in figure 8 . We see the very strong stabilization which takes place at a rather small value of the Prandtl number, denoted in a general way as $\operatorname{Pr}_{t}$ and specifically for $T a_{m}=0$ as $\operatorname{Pr}_{t, 0}\left(\operatorname{Pr}_{t, 0} \approx 3 \times 10^{-4}\right)$. This stabilization effect at low $P r$ was already found for a horizontal cylinder of rectangular crosssection (Lyubimova et al. 2009). It was related to the presence of a stable temperature 


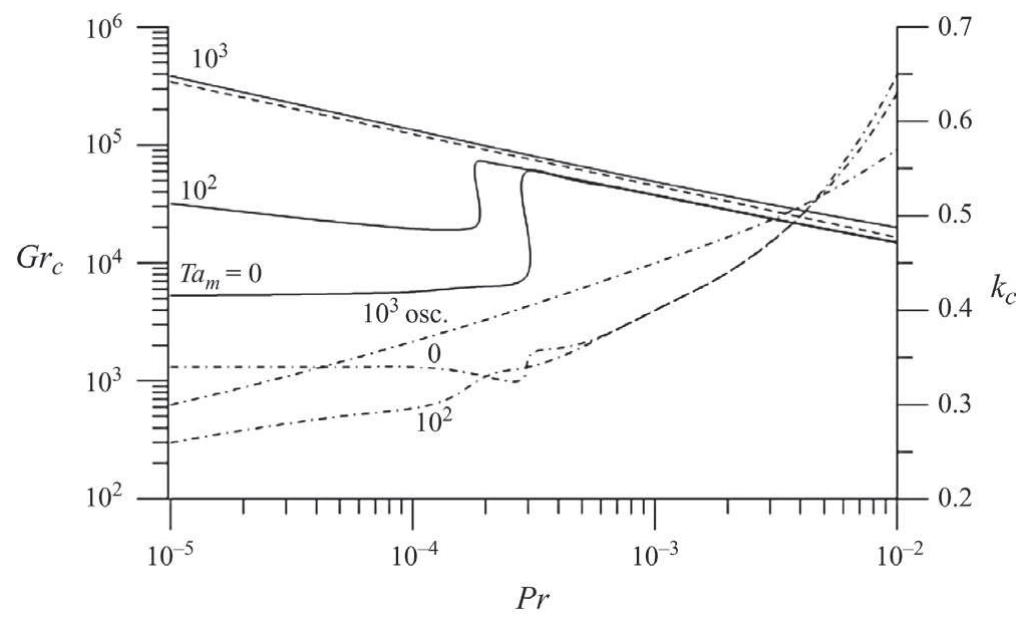

FIGURE 8. Critical Grashof number, $G r_{c}$, as a function of the Prandtl number $P r$ for three values of $\operatorname{Ta}_{m}\left(T a_{m}=0,100,1000\right)$. Solid curves indicate steady thresholds and dashed curves oscillatory thresholds. The corresponding critical wavenumbers $k_{c}$ (dash-dotted curves) are given for the dominant modes.

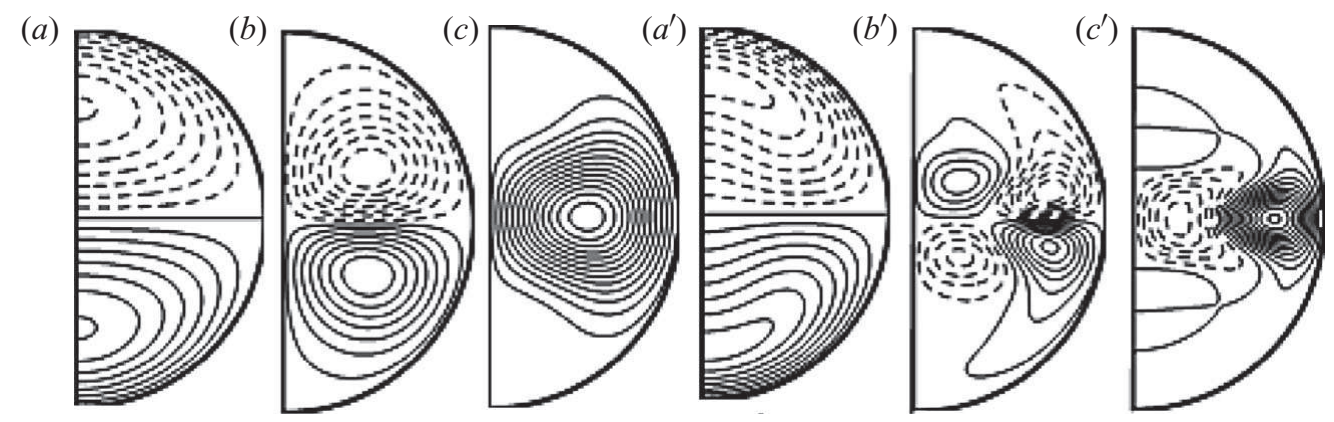

FIGURE 9. Isolines of the longitudinal velocity of the basic flow $\left(a, a^{\prime}\right)$, and real $\left(b, b^{\prime}\right)$ and imaginary $\left(c, c^{\prime}\right)$ parts of the longitudinal velocity perturbation, at the critical threshold for $P r=0.00029\left(G r_{c}=7415, k_{c}=0.33\right)(a-c)$ and $\operatorname{Pr}=0.0003\left(G r_{c}=60756, k_{c}=0.35\right)\left(a^{\prime}-c^{\prime}\right)$ in a situation without magnetic field $\left(\mathrm{Ta}_{m}=0\right)$. These cases are just below and above the domain of sharp stabilization, respectively. Because of the symmetry properties, only half of the cross-section is shown.

stratification in the region where the perturbations developed, at the interface between the counterflows. Note that the critical curve evolves slightly backwards close to $\operatorname{Pr}_{t, 0}$, which indicates the presence of a slight hysteresis in this zone. Concerning the critical wavenumber, after a slight decrease, it also abruptly increases at $\operatorname{Pr}=\operatorname{Pr}_{t, 0}$. Beyond $P r_{t, 0}$, for $\operatorname{Pr}_{t, 0}<\operatorname{Pr}<0.01$, the critical Grashof number decreases as $\operatorname{Pr}$ is increased, whereas the wavenumber increases up to $k_{c} \approx 0.63$. The decrease of the critical threshold in this domain of $P r$ can be well approximated by a scaling law, $G r_{c} \sim \mathrm{Pr}^{-2 / 5}$. A further increase of the Prandtl number will result in a stabilization effect still connected to the stable temperature stratification (Lyubimova et al. 2009).

Figure 9 shows the basic flow (isolines of the longitudinal velocity $W_{0}$ ) and the critical perturbation (real and imaginary parts of the $w$ eigenvector) at Prandtl number values close to $\operatorname{Pr}_{t, 0}$, below and above the sharp stabilization. Below the 
(a)

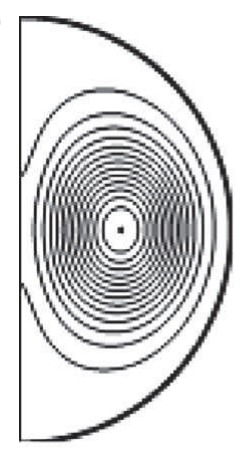

(b)

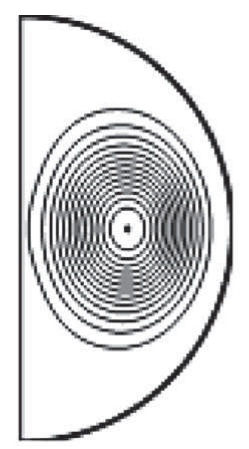

(c)

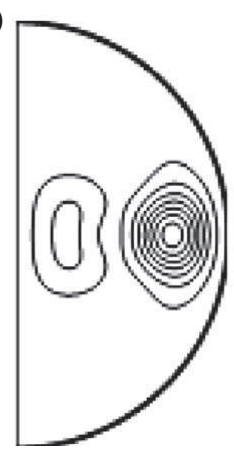

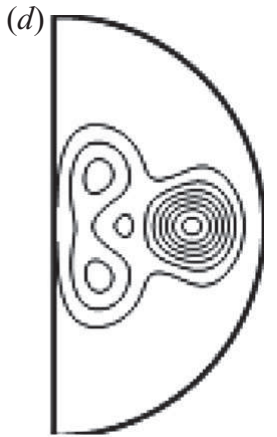

FIGURE 10. Perturbation kinetic energy at the critical threshold in situations without magnetic field $\left(T a_{m}=0\right)$ for ( $\left.a\right) \operatorname{Pr}=0\left(G r_{c}=5263\right),(b) P r=0.00029\left(G r_{c}=7415\right),(c) P r=0.0003$ $\left(G r_{c}=60756\right)$ and $(d) \operatorname{Pr}=0.02\left(G r_{c}=11644\right)$. Because of the symmetry properties, only half of the cross-section is shown.

stabilization $\left(P r=0.00029, G r_{c}=7415, k_{c}=0.33\right)$ (figure $\left.9 a-c\right)$, the basic flow and the critical perturbation are nearly the same as those obtained at zero Prandtl number (compare with figures $2 b$ and $7 c$ ). Above the stabilization $\left(\mathrm{Pr}=0.0003, G r_{c}=60756\right.$, $k_{c}=0.35$ ) (figures $9 a^{\prime}, b^{\prime}, c^{\prime}$ ), the results are quite different. For the basic flow, we see an influence of the cross-section vortices on the longitudinal velocity $W_{0}$, similarly to what was shown in figure $2\left(b^{\prime}\right)$, and for the perturbation, we see that the different peaks of the $w$ perturbation have split into two, the strongest peaks being close to the lateral boundary. This change can also be clearly identified on the perturbation kinetic energy plotted for $\operatorname{Pr}=0,0.00029,0.0003$ and 0.02 in figure 10. (Note that the kinetic energy is defined by $0.5\left(v \cdot v^{*}\right)=0.5\left(v_{r}^{2}+v_{i}^{2}\right)$, so that it is positive and appears in the cross-section as symmetric with respect to both horizontal and vertical middle axis. The breaking of the $S_{l}$ symmetry, however, indicates that only the velocity perturbation along the $x$-direction is non-zero in the $V_{l}$ plane.) For $\operatorname{Pr}=0.00029$, we have two strong peaks of kinetic energy on both sides of the $V_{l}$ plane, each being centred in one half of the cylinder (this is very similar to what is obtained at $\operatorname{Pr}=0$ ), whereas for $P r=0.0003$, each peak has given two peaks, the main peak being close to the boundary. The kinetic energy is then not much changed for larger $\operatorname{Pr}$ values, as it can be seen from the plot at $P r=0.02$. All this indicates a change in the instability mode at $\operatorname{Pr}_{t, 0}$. This looks quite similar to what was observed in the case of a horizontal cylinder of rectangular cross-section (Lyubimova et al. 2009), where a new sidewall mode was identified beyond the stabilization at $\operatorname{Pr}_{t, 0}$. Note that for $\operatorname{Pr}<P r_{t, 0}$, only one peak of kinetic energy, centred in the cross-section, was found for a rectangular cross-section, whereas we have two peaks for a circular cylinder: this is due to the fact that the $S_{l}$ symmetry is broken in the second case and preserved in the former case. Finally note that, as illustrated in figure 9, the symmetry properties described for $\operatorname{Pr}=0$ remain the same on the whole range of $\operatorname{Pr}$ values, even in the domain beyond the transition at $P r_{t, 0}$.

In order to better characterize the changes which occur when the Prandtl number is increased, an energy analysis was performed for different values of $\operatorname{Pr}(\operatorname{Pr}=0$, $0.00029,0.0003$ and 0.02 ). The perturbation kinetic energy budget corresponding to (4.8) is shown in table 1. For the four values of $P r$, we see that the buoyancy contribution is really negligible and that the destabilization comes from the shear of the basic flow and is related to the terms $E_{s x}^{\prime}$ and $E_{s y}^{\prime}$ (connected to the gradients 


\begin{tabular}{ccccc}
\hline & $P r=0$ & $P r=0.00029$ & $P r=0.0003$ & $P r=0.02$ \\
$T a_{m}=0$ & $G r_{c}=5263$ & $G r_{c}=7415$ & $G r_{c}=60756$ & $G r_{c}=11644$ \\
$E_{b}^{\prime}$ & $k_{c}=0.34$ & $k_{c}=0.33$ & $k_{c}=0.35$ & $k_{c}=0.77$ \\
$E_{s}^{\prime}$ & 0 & $-2 \times 10^{-4}$ & $-8 \times 10^{-5}$ & $-3.9 \times 10^{-3}$ \\
$E_{s x}^{\prime}$ & 1 & $\approx 1$ & $\approx 1$ & $\approx 1$ \\
$E_{s y}^{\prime}$ & 0.157 & 0.134 & 0.263 & 0.506 \\
& 0.843 & 0.865 & 0.735 & 0.470
\end{tabular}

TABLE 1. Kinetic energy budgets associated with the hydrodynamic modes at threshold for different values of the Prandtl number in the case without RMF $\left(T a_{m}=0\right)$. For $\operatorname{Pr}=0, E_{s x}^{\prime}$ and $E_{s y}^{\prime}$ are the only shear contributions. For $P r=0.00029$ and 0.0003 (below and above the transition at $P r_{t, 0}$, respectively) and $P r=0.02$, the other contributions exist but are small. (The largest is connected to $\left(\partial U_{0} / \partial x\right)$ and its values are $5.9 \times 10^{-4}, 1.15 \times 10^{-3}$ and $2.18 \times 10^{-2}$, respectively.)
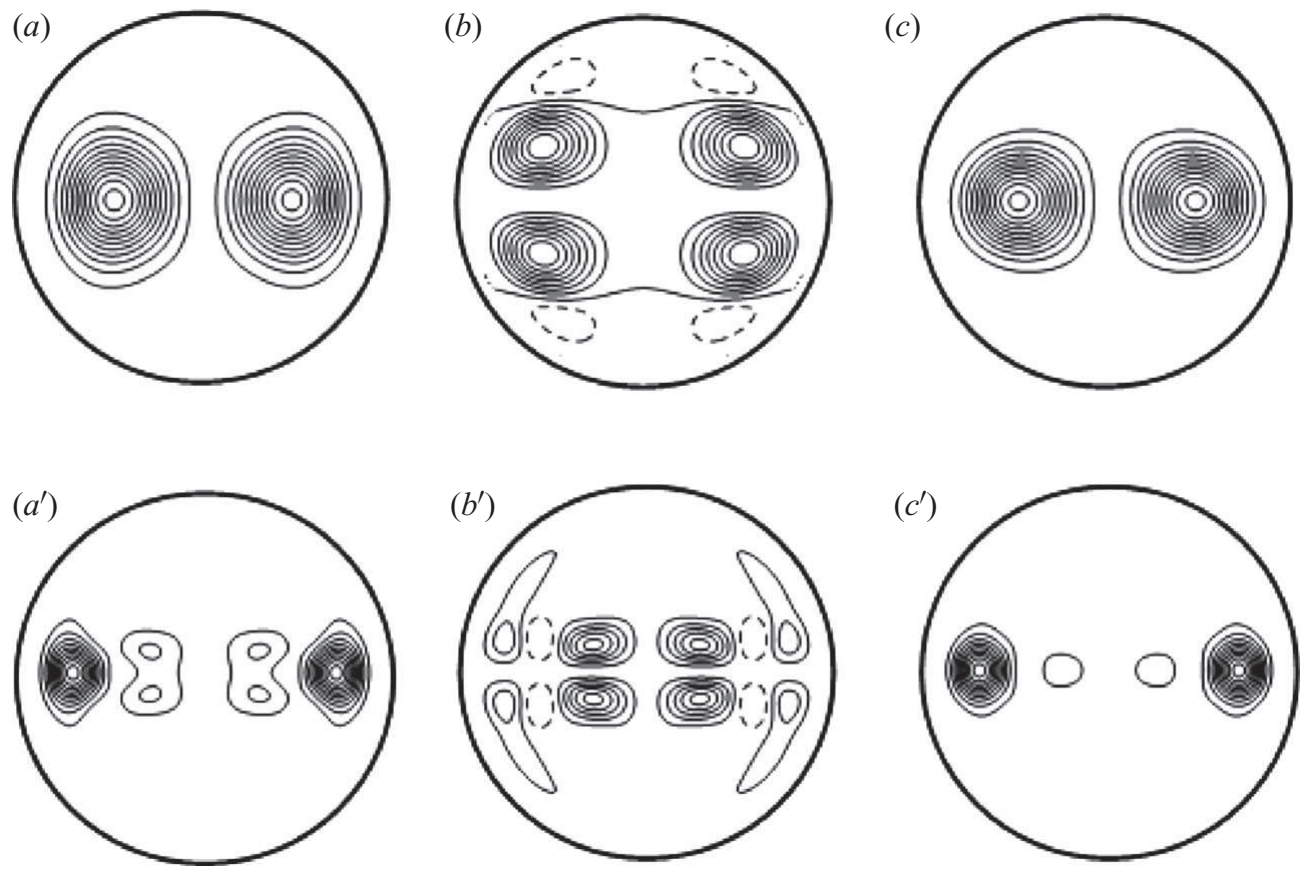

FIGURE 11. Isolines of the shear energy field $\mathscr{E}_{s}^{\prime}\left(a, a^{\prime}\right)$ and of its main contributions $\mathscr{E}_{s x}^{\prime}\left(b, b^{\prime}\right)$ and $\mathscr{E}_{s y}^{\prime}\left(c, c^{\prime}\right)$ at the critical threshold for $\operatorname{Pr}=0.00029\left(G r_{c}=7415\right)(a-c)$ and $\operatorname{Pr}=0.0003$ $\left(G r_{c}=60756\right)\left(a^{\prime}-c^{\prime}\right)$ in a situation without magnetic field $\left(T a_{m}=0\right)$.

of the longitudinal velocity $W_{0}$ ) which dominate the other shear terms. As in the case of the rectangular cross-section (Lyubimova et al. 2009), the main shear term is generally $E_{s y}^{\prime}$ (connected to the vertical gradient of the longitudinal velocity, $\partial W_{0} / \partial y$ ), but $E_{s x}^{\prime}$ is not negligible in our case and even becomes slightly dominant for $\operatorname{Pr}=0.02$.

The analysis of the shear terms is presented in figures 11 and 12 for both $\operatorname{Pr}=$ 0.00029 and 0.0003. $(P r=0.00029$ is representative of the cases before the transition 

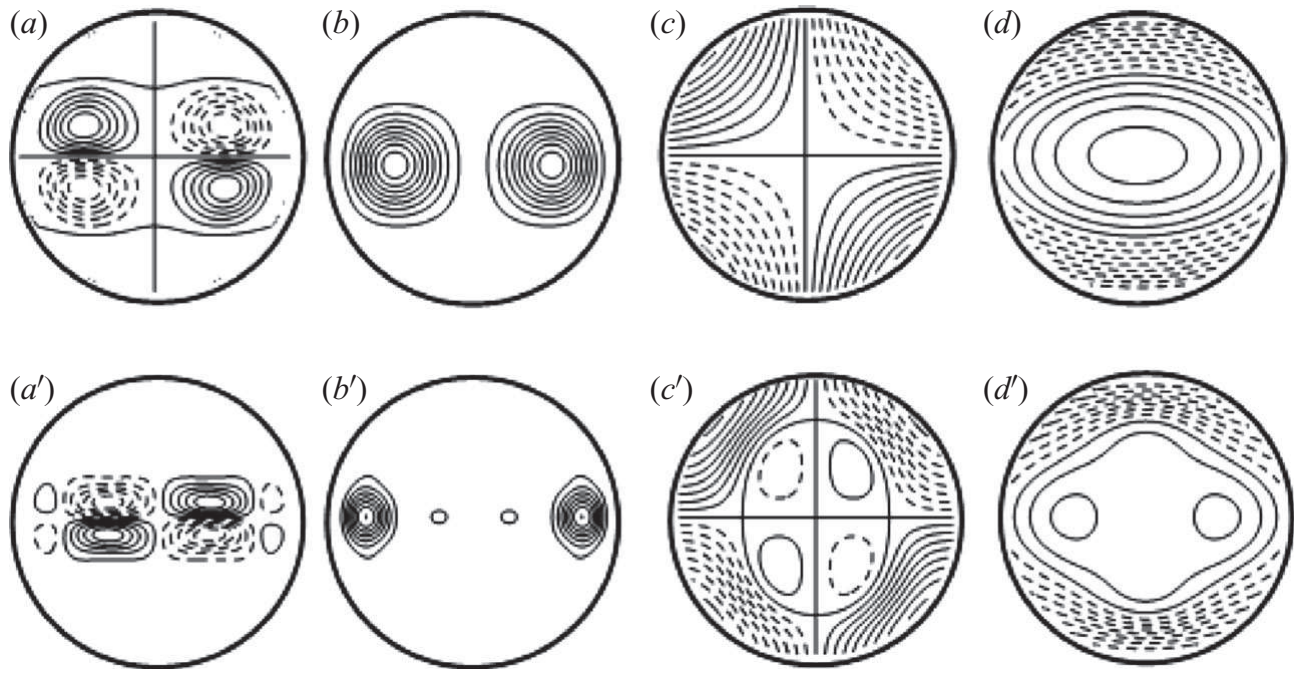

FIGURE 12. Isolines of the velocity disturbance products $F_{x}\left(a, a^{\prime}\right)$ and $F_{y}\left(b, b^{\prime}\right)$ and of the basic flow shears $M_{x}\left(c, c^{\prime}\right)$ and $M_{y}\left(d, d^{\prime}\right)$ (which are such that $\mathscr{E}_{s x}^{\prime}=M_{x} F_{x}$ and $\mathscr{E}_{s y}^{\prime}=M_{y} F_{y}$ ) at the critical threshold for $\operatorname{Pr}=0.00029\left(G r_{c}=7415\right)(a-d)$ and $\operatorname{Pr}=0.0003\left(G r_{c}=60756\right)$ $\left(a^{\prime}-d^{\prime}\right)$ in a situation without magnetic field $\left(T a_{m}=0\right)$.

at $\operatorname{Pr}_{t, 0}$ (as $P r=0$ ), and $P r=0.0003$ is representative of the cases beyond $P r_{t, 0}$.) We first give the plots of the global shear energy field $\mathscr{E}_{s}^{\prime}$ and its main contributions $\mathscr{E}_{s x}^{\prime}$ and $\mathscr{E}_{s y}^{\prime}$ in figure 11 . We then show the terms which build these shear contributions, $M_{x}$ and $F_{x}$ for $\mathscr{E}_{s x}^{\prime}$ and $M_{y}$ and $F_{y}$ for $\mathscr{E}_{s y}^{\prime}$, in figure 12. In figure $11(a-c)$, we see that for $\operatorname{Pr}=0.00029$ the global shear energy field $\mathscr{E}_{s}^{\prime}$ presents two wide peaks which develop along the $y$-axis, i.e. at the border between the counterflows. These peaks mainly reflect the influence of $\mathscr{E}_{s y}^{\prime}$, slightly reinforced by $\mathscr{E}_{s x}^{\prime}$. As shown in figure $12(a-d), \mathscr{E}_{s y}^{\prime}$ is generated by the two positive peaks of the velocity disturbance product $F_{y}$ which appear in a large zone of positive values of the basic flow shear $M_{y}$. For $\mathscr{E}_{s x}^{\prime}$, the four peaks, with different signs, of $F_{x}$ are generated in zones of $M_{x}$ with the same sign. For $\mathrm{Pr}=0.0003$, in contrast, $\mathscr{E}_{s}^{\prime}$ has six peaks (figure $11 a^{\prime}-c^{\prime}$ ). The two main peaks are still along the $y$-axis, but they are narrower than for $P r=0.00029$ and located closer to the boundaries. These peaks reflect the influence of $\mathscr{E}_{s y}^{\prime}$. The four smaller peaks are closer to the centre and on both sides of the $y$-axis, and they reflect the influence of $\mathscr{E}_{s x}^{\prime}$. As shown in figure $12\left(a^{\prime}-d^{\prime}\right), \mathscr{E}_{s y}^{\prime}$ is generated by the growth of two positive peaks, closer to the boundaries, in the velocity disturbance product $F_{y}$. These peaks appear in two zones where we now have extrema of the basic flow shear $M_{y}$, in connection with the modification of the longitudinal flow observed in figure $9\left(a^{\prime}\right)$. The four main peaks of $\mathscr{E}_{s x}^{\prime}$ are also different from those obtained for $\mathrm{Pr}=0.00029$, as both the velocity disturbance product $F_{x}$ and the basic flow shear $M_{x}$ (in the central zone where the peaks appear) have changed.

All this confirms that the instabilities are due to the shear which develops at the boundary between the counterflows. For $P r<P r_{t, 0}$, the instability rather grows near the centre where the shear is dominant, but we have two separated peaks because of the $S_{l}$ symmetry breaking. For $P r>P r_{t, 0}$, the instability develops closer to the sidewalls in the region where the shear becomes dominant because of the influence of the counter-rotating vortices on the longitudinal basic flow. The strong stabilization 


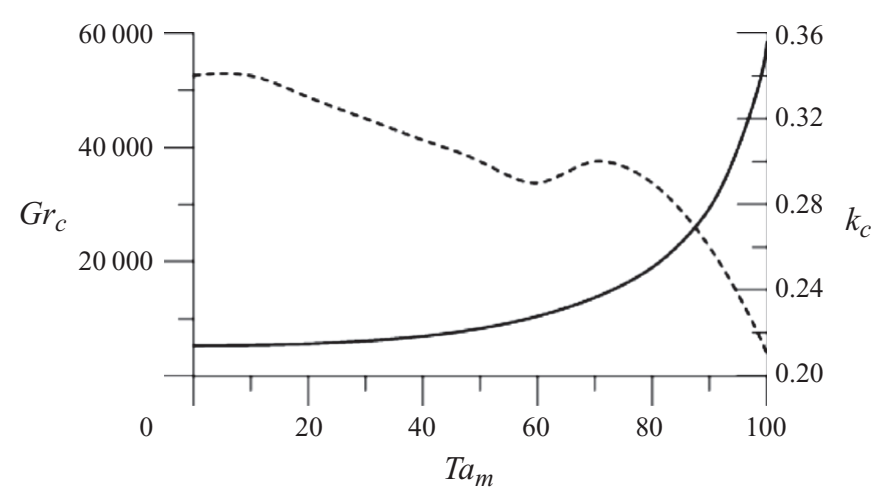

FIGURE 13. Critical Grashof number $G r_{c}$ (solid line) and wavenumber $k_{c}$ (dashed line) as a function of the magnetic Taylor number $\mathrm{Ta}_{m}$ for the more dangerous hydrodynamic mode at $\operatorname{Pr}=0$.

at $\operatorname{Pr}_{t, 0}$ then corresponds to the transition between the usual shear instability of counterflows and a new sidewall shear instability.

5.2. The effect of an RMF on convective flow stability: zero Prandtl number case When the RMF is applied, the basic flow at zero Prandtl number is given by (3.13) and (3.15). We thus have

$$
\begin{gathered}
\boldsymbol{U}_{0}(r, \alpha)=\frac{1}{8} T a_{m}\left(1-r^{2}\right) r \boldsymbol{e}_{\alpha}, \\
W_{0}(r, \alpha)=\operatorname{Gr}\left(\mathscr{W}_{r}(r) \sin \alpha+\mathscr{W}_{i}(r) \cos \alpha\right),
\end{gathered}
$$

where $\mathscr{W}(r)=\mathscr{W}_{r}(r)+\mathrm{i} \mathscr{W}_{i}(r)$ is the solution of (3.16) with boundary condition (3.17). The basic solution corresponds to circular streamlines in the cross-section and to the longitudinal velocity shown in figure 4.

As explained in $\S 3.3$, the symmetry group of the general problem with an RMF is generated by $T_{z_{0}}$ and $S_{c}$. Here also, each eigenmode with finite wavelength $\left(k_{c} \neq 0\right)$ breaks the translation invariance and is thus associated with a bifurcation which produces an infinite number of equivalent steady states parametrized by $z_{0}$. Each eigenmode possesses points of symmetry (separated by half a wavelength) on the $z$-axis and points of antisymmetry in between. By continuity with the case without an $\mathrm{RMF}$, the point at $z=0$ will be a point of antisymmetry and the point at $z=\lambda / 4$ will be a point of symmetry. According to these symmetries, $X_{r}$, which is the perturbation in the cross-section at $z=0$, will appear antisymmetric with respect to the central point of the cross-section (at $x=0$ and $y=0$ ), whereas $X_{i}$, which is the perturbation in the cross-section at $z=\lambda / 4$, will appear symmetric. In intermediate cross-sections, the perturbation will not present any symmetry with respect to the central point. We have checked these symmetry properties of the critical eigenvectors, which are also valid for non-zero $\mathrm{Pr}$. The perturbations (calculated for example at the critical threshold for $\left.T a_{m}=100\left(G r_{c}=58357, k_{c} \approx 0.21\right)\right)$ appear as the evolution under the RMF effect of those obtained at $T a_{m}=0$, except that the $w$ perturbations have greatly evolved.

The stability curve showing the dependence of the critical Grashof number $G r_{c}$ (for the more dangerous steady hydrodynamic mode) on the magnetic Taylor number $T a_{m}$ for $\operatorname{Pr}=0$ and the curve giving the corresponding variation of the critical wavenumber $k_{c}$ are shown in figure 13. We see a steep monotonic growth of $G r_{c}$ with $T a_{m}$ which demonstrates the very strong stabilization induced by the RMF at $\operatorname{Pr}=0$. 


\begin{tabular}{ccccc}
\hline & $T a_{m}=0$ & $T a_{m}=50$ & $T a_{m}=80$ & $T a_{m}=110$ \\
$\operatorname{Pr}=0$ & $k_{c}=5263$ & $G r_{c}=8291$ & $G r_{c}=18966$ & $G r_{c}=246867$ \\
$E_{b}^{\prime}=0.34$ & $k_{c}=0.3$ & $k_{c}=0.29$ & $k_{c}=0.14$ \\
$E_{s}^{\prime}$ & 0 & 0 & 0 & 0 \\
$E_{s x}^{\prime}$ & 1 & $\approx 1$ & $\approx 1$ & $\approx 1$ \\
$E_{s y}^{\prime}$ & 0.157 & 0.788 & 1.831 & 2.611 \\
& 0.843 & 0.212 & -0.831 & -1.611
\end{tabular}

TABLE 2. Kinetic energy budgets associated with the hydrodynamic modes at threshold for different values of the magnetic Taylor number at zero Prandtl number. For $T a_{m}=0, E_{s x}^{\prime}$ and $E_{s y}^{\prime}$ are the only shear contributions. For the other values of $T a_{m}$, the other contributions exist but are very small (smaller than $10^{-3}$ ).
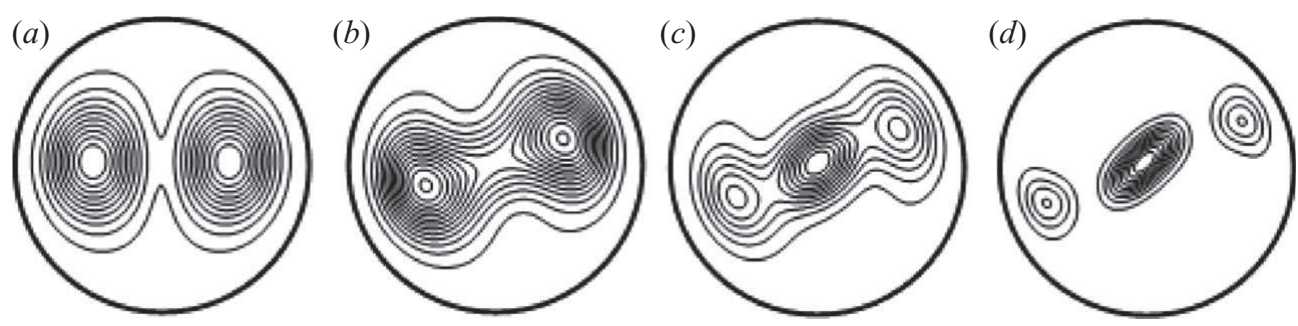

Figure 14. Perturbation kinetic energy at the critical threshold for $\operatorname{Pr}=0$ and different values of the magnetic Taylor number $T a_{m}:(a) T a_{m}=0\left(G r_{c}=5263\right),(b) T a_{m}=50\left(G r_{c}=8291\right)$, (c) $T a_{m}=80\left(G r_{c}=18966\right)$ and $(d) T a_{m}=110\left(G r_{c}=246867\right)$.

This growth of $G r_{c}$ can be quite well fitted by an exponential law: $G r_{c} / G r_{c}\left(T a_{m}=\right.$ $0) \propto \exp \left(1.5 \times 10^{-5} \mathrm{Ta}_{m}^{2.6}\right)$. The critical wavenumber $k_{c}$ globally decreases as $T a_{m}$ is increased. This evolution is, however, non-monotonous: a slight initial increase is found near $T a_{m}=0$ and another increase in the range $60 \leqslant T a_{m} \leqslant 70$.

The change with $T a_{m}$ of the perturbation kinetic energy at the critical threshold for $\operatorname{Pr}=0$ is depicted in figure 14. The four chosen values of $T a_{m}\left(T a_{m}=0,50\right.$, $80,110)$ correspond to increasing values of the critical Grashof number $\left(G r_{c}=5263\right.$, 8291, 18966 and 246867, respectively). (Note that for $T a_{m} \neq 0$, the kinetic energy appears as symmetric with respect to the central point of the cross-section only.) We see that, with the increase of $T a_{m}$, the two peaks obtained at $T a_{m}=0$ begin to tilt in the direction of rotation of the magnetic field. There is also an increase of the kinetic energy at the centre of the cross-section which eventually gives a central peak. At $T a_{m}=80$, this central peak already dominates the two other peaks which are shifted closer to the boundaries. Finally, at $T a_{m}=110$, the dominant central peak is isolated from the two other peaks whose contribution to the kinetic energy will decrease as $T a_{m}$ is further increased. Other calculations performed at fixed values of $G r$ and $k$ $(G r=5000, k=0.3)$ for increasing values of $T a_{m}$ have shown a similar increase of a central peak which eventually dominates the kinetic energy of the dominant eigenmode. This confirms that the growth of a central peak in the kinetic energy is really connected to the influence of the RMF.

In order to better characterize the changes which occur when $T a_{m}$ is increased at $P r=0$, energy analyses were performed. The perturbation kinetic energy budgets for the same cases as in figure 14 are shown in table 2 . There is no buoyancy contribution in these cases, and the destabilization still comes from the shear energy terms $E_{s x}^{\prime}$ 

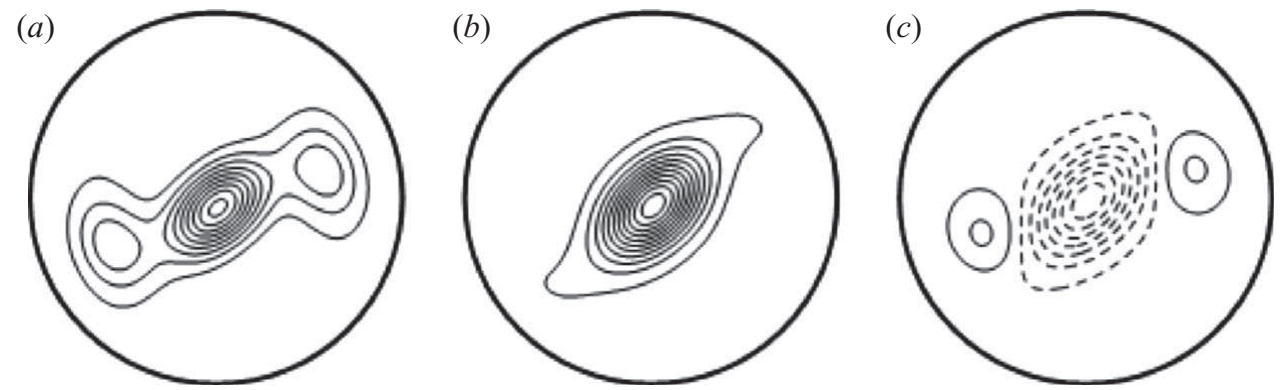

FIGURE 15. Isolines of the shear energy field $\mathscr{E}_{s}^{\prime}(a)$ and of its main contributions $\mathscr{E}_{s x}^{\prime}(b)$ and $\mathscr{E}_{s y}^{\prime}(c)$ at the critical threshold $\left(G r_{c}=18966\right)$ for $T a_{m}=80$ and $\operatorname{Pr}=0$.
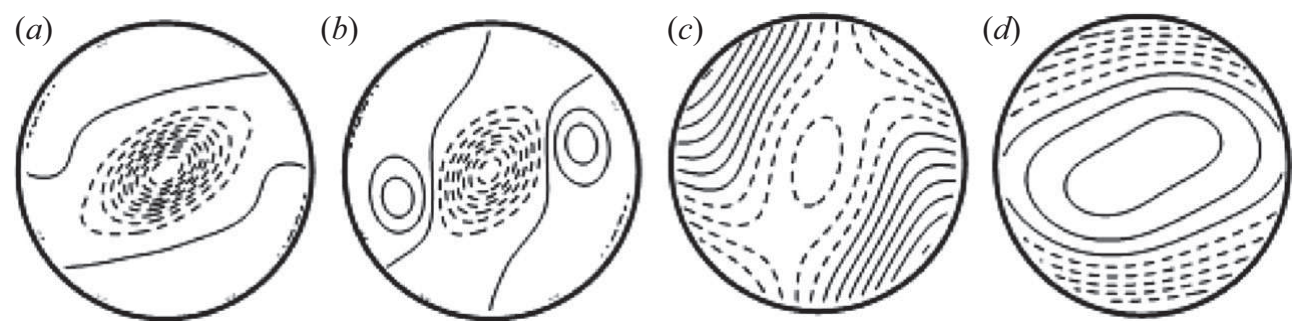

FIGURE 16. Isolines of the velocity disturbance products $F_{x}(a)$ and $F_{y}(b)$ and of the basic flow shears $M_{x}(c)$ and $M_{y}(d)$ (which are such that $\mathscr{E}_{s x}^{\prime \prime}=M_{x} F_{x}$ and $\mathscr{E}_{s y}^{\prime}=M_{y} F_{y}$ ) at the critical threshold $\left(G r_{c}=18966\right)$ for $T a_{m}=80$ and $P r=0$.

and $E_{s y}^{\prime}$. Strong changes, however, affect the respective contributions of these two terms when $T a_{m}$ is increased. $E_{s y}^{\prime}$ which is the dominant destabilizing term at $T a_{m}=0$ decreases as $T a_{m}$ is increased, and even quickly becomes negative, giving a stabilizing contribution. $E_{s x}^{\prime}$, in contrast, gives a destabilizing contribution which increases with $T a_{m}$ and becomes quickly dominant.

The analysis of the shear terms is presented in figures 15 and 16 for $T a_{m}=80$. The global shear energy field $\mathscr{E}_{s}^{\prime}$ in figure 15 looks similar to the corresponding kinetic energy field (figure 14c) with three non-disjoined peaks, along a diameter slightly inclined with respect to the horizontal. The two weaker non-central peaks reflect the influence of the two positive peaks of $\mathscr{E}_{s y}^{\prime}$ and they correspond to the evolution of the two peaks found at $T a_{m}=0$. A negative peak has, however, appeared in $\mathscr{E}_{s y}^{\prime}$ near the centre, but this peak is dominated by the positive central peak of $\mathscr{E}_{s x}^{\prime}$. As shown in figure 16 , the positive peak of $\mathscr{E}_{s x}^{\prime}$ is generated by a large negative peak in the velocity disturbance product $F_{x}$, around the centre, in a zone where the basic flow shear $M_{x}$ is now negative. The peaks of $\mathscr{E}_{s y}^{\prime}$ are generated by similar peaks in the velocity disturbance product $F_{y}$ (a central negative peak and two positive peaks closer to the boundaries) in a large zone where the basic flow shear $M_{y}$ is positive.

The positive peak which appears in the perturbation kinetic energy when $T a_{m}$ is increased is then principally connected to the modifications which affect the shear energy $\mathscr{E}_{s x}^{\prime}$ under the influence of the RMF: transverse gradients of the longitudinal velocity appear around the centre of the cavity, which generate velocity perturbations in this zone, thus leading to a destabilizing shear contribution. The modifications of $\mathscr{E}_{s y}^{\prime}$ also play a role: the appearance of negative values in the velocity disturbance product $F_{y}$, near the centre, will moderate the destabilizing influence of $\mathscr{E}_{s x}^{\prime}$, and will lead to the shift of the destabilizing peaks of $\mathscr{E}_{s y}^{\prime}$ closer to the boundaries. 


\subsection{The effect of an RMF on convective flow stability: non-zero Prandtl numbers}

We now investigate the effect of the RMF on the convective flow stability for non-zero $\mathrm{Pr}$ values. For that, the dependence of the critical Grashof number on the Prandtl number is calculated for two values of the magnetic Taylor number, $T a_{m}=100$ and $T a_{m}=1000$. Figure 8 depicts these stability curves and the corresponding wavenumbers, together with those obtained for $T a_{m}=0$. For $T a_{m}=0$ and 100 , only the steady thresholds, which correspond to the more dangerous perturbations, are given. For $T a_{m}=1000$, both steady and oscillatory thresholds are given. First note that the thresholds increase with the increase of $T a_{m}$, but that this increase strongly depends on the value of $P r$. The sharp increase of the thresholds found for $T a_{m}=0$ at $\operatorname{Pr}_{t, 0}$ and corresponding to a transition between two different hydrodynamic modes is still present for $T a_{m}=100$. It occurs, however, at a smaller value of $P r_{t}$, and the amplitude of the increase is reduced. In the range of $\operatorname{Pr}$ below the transition at $\operatorname{Pr}_{t}$, the stability curve for $T a_{m}=100$ shows a decrease of the thresholds as $\operatorname{Pr}$ is increased. For example, we have $G r_{c}=58357$ for $\operatorname{Pr}=0$ and $G r_{c}=20113$ for $\operatorname{Pr}=0.00018$, which indicates a clear decrease of the stabilizing effect of the magnetic field as $\mathrm{Pr}$ is increased in this $\mathrm{Pr}$ range. For $\mathrm{Pr}$ values above the transition, the stability curve for $T a_{m}=100$ almost follows the critical curve obtained for $T a_{m}=0$ for the sidewall modes, which indicates that the RMF has only a very weak stabilizing effect on these sidewall modes. For larger values of $T a_{m}$, the value of $P r_{t}$ will still decrease, leading to the extension towards smaller $P r$ values of the critical curve related to the sidewall modes. Finally, for $T a_{m}=1000$, the transition at $P r_{t}$ is no more visible in the $\mathrm{Pr}$ range studied $\left(10^{-5} \leqslant P r \leqslant 0.01\right)$, and only the critical curve portion related to the sidewall modes is visible. In fact, for $T a_{m}=1000$, the steady modes are no more, the more dangerous modes and an oscillatory critical curve slightly precedes the steady curve. Concerning the critical wavenumbers, for $T a_{m}=100$ they increase with $\mathrm{Pr}$, but are still influenced by the strong increase of the thresholds around $\operatorname{Pr}_{t}$ and then almost follow the wavenumber curve obtained for $T a_{m}=0$ in the domain of the sidewall instability. For $T a_{m}=1000$, the critical wavenumber curve increases more regularly in the whole $\operatorname{Pr}$ range studied.

We now analyse the basic flow and the critical perturbations obtained in the presence of an RMF ( $\left.T a_{m}=100\right)$ for two Prandtl number values close to $P r_{t}$, below and above the transition. Below the transition $\left(P r=0.00018, G r_{c}=20113, k_{c}=0.32\right)$, the basic flow (figure $17 a-c$ ) looks similar to that obtained for $\operatorname{Pr}=0$. The inclination of the longitudinal velocity is slightly weaker than for $\mathrm{Pr}=0$ (compare with figure 4 for $T a_{m}=100$ ). The streamlines of the transverse flow are also not completely circular as they were for $P r=0$. These streamlines can be quite well predicted by the analytical expression (3.33) derived in the limit case of small values of $T a_{m}$ and $\operatorname{Pr}$ (the case at $\operatorname{Pr}=0.00018$ corresponds to $a \approx 16$ and can be compared with the results for $a=5$ and $a=100$ presented in figure 5). Finally the temperature field in the cross-section is quite similar to the longitudinal flow field, with a similar inclination due to the RMF. The critical perturbations for $P r=0.00018$ can still be seen as the rotation of those obtained without a magnetic field for $P r<P r_{t, 0}$ (figure 7). They are also close to those obtained for $\operatorname{Pr}=0$ at $T a_{m}=100$, but with a slightly different orientation of the patterns connected to the modification of the basic flow. The largest changes affect the patterns for the $w$ perturbation.

Above the transition $\left(\mathrm{Pr}=0.00019, G r_{c}=73664, k_{c}=0.33\right)$, the basic flow (figure $17 a^{\prime}-c^{\prime}$ ) seems to be unaffected by the RMF. Except for the $S_{l}$ symmetry which is no more valid, the flow structure is really similar to that obtained at $T a_{m}=0$ 


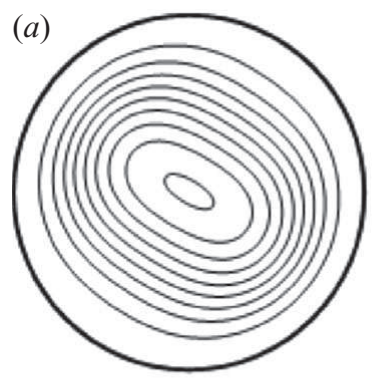

(b)

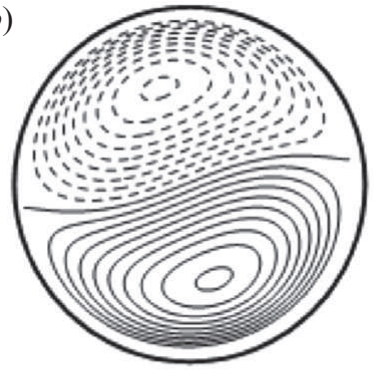

$\left(a^{\prime}\right)$

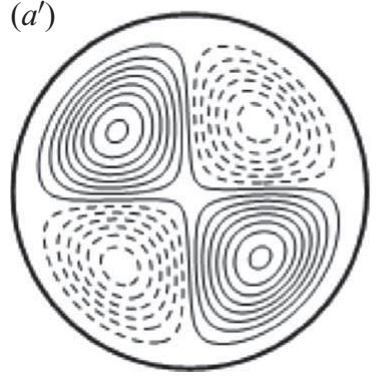

$\left(b^{\prime}\right)$

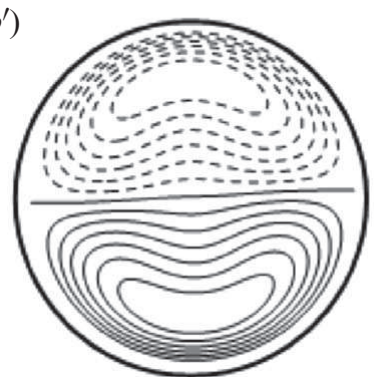

(c)

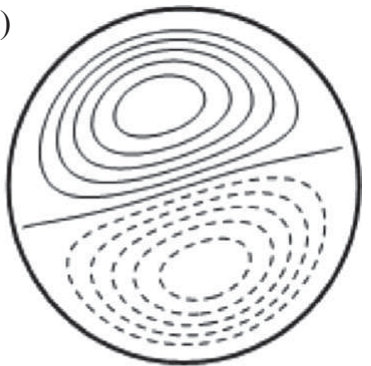

$\left(c^{\prime}\right)$

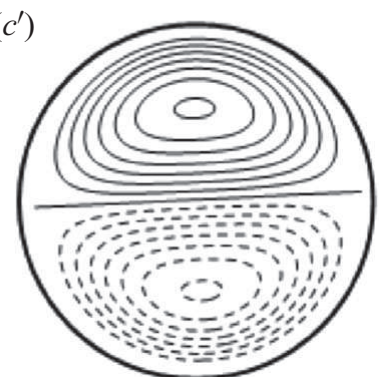

FIGURE 17. Streamlines of the transverse flow $\left(a, a^{\prime}\right)$, isolines of the longitudinal velocity $\left(b, b^{\prime}\right)$ and isolines of the temperature field $\left(c, c^{\prime}\right)$ for the basic solution at threshold for $T a_{m}=100$ in two cases just below and above the domain of sharp stabilization: $\operatorname{Pr}=0.00018\left(G r_{c}=20113\right)$ $(a-c)$ and $P r=0.00019\left(G r_{c}=73664\right)\left(a^{\prime}-c^{\prime}\right)$.
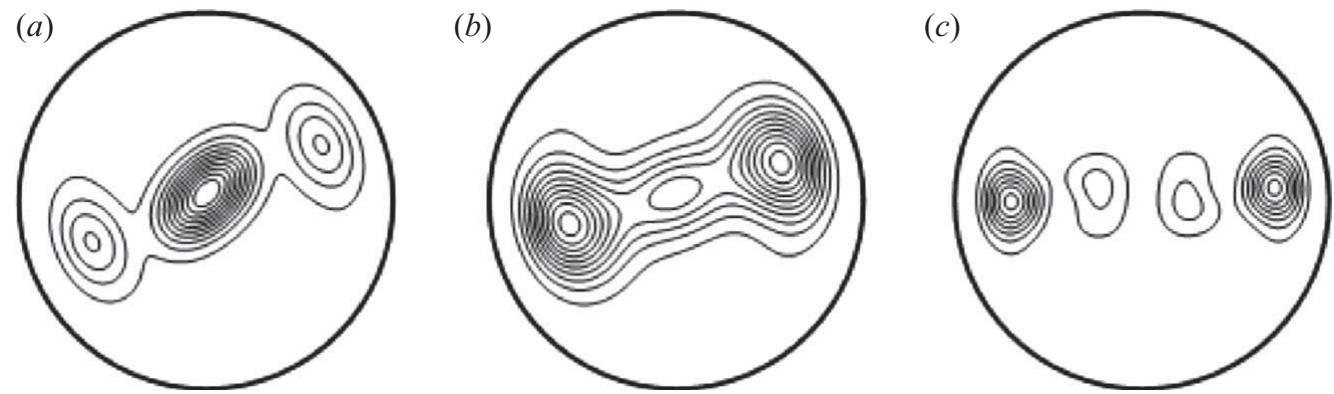

FIGURE 18. Perturbation kinetic energy at the critical threshold in situations with RMF $\left(T a_{m}=100\right)$ for $(a) \operatorname{Pr}=0\left(G r_{c}=58357\right),(b) \operatorname{Pr}=0.00018\left(G r_{c}=20113\right)$ and $(c)$ $\operatorname{Pr}=0.00019\left(G r_{c}=73664\right)$.

for $\operatorname{Pr}>\operatorname{Pr}_{t, 0}$ (compare with figure $2 b^{\prime}$ ), with a strong influence of the cross-section vortices on the longitudinal flow. The structure of the critical perturbations for $\operatorname{Pr}=0.00019$, despite different symmetry properties, is also close to the structure obtained for $P r>P r_{t, 0}$ in the absence of a magnetic field (figure $9 b^{\prime}, c^{\prime}$ ), which still confirms that for $\operatorname{Pr}>\operatorname{Pr}_{t}$ the effect of the RMF at $T a_{m}=100$ is nearly negligible.

The perturbation kinetic energy plotted for different values of $\operatorname{Pr}$ at $T a_{m}=100$ in figure 18 illustrates how the modifications induced by the RMF evolve with Pr. Below the transition (figure 18a,b), we find three peaks aligned along a diameter which is inclined with respect to the horizontal, as already mentioned for $\operatorname{Pr}=0$ and 
non-zero $\mathrm{Ta}_{m}$ in $\S 5.2$. The increase of $\operatorname{Pr}$ in this $\operatorname{Pr}$ range decreases the inclination of the peak structure. It also decreases the intensity of the central peak (connected to the RMF) with respect to the two other peaks (already existing at $T a_{m}=0$ ). Above the transition (figure 18c), we find four peaks which are really similar to those obtained at $T a_{m}=0$ (figure $10 c$ ), except that they are less symmetrical.

We finally present the energy analysis results in cases with an RMF $\left(T a_{m}=100\right)$ for different values of $\operatorname{Pr}(\operatorname{Pr}=0,0.00018$ and 0.00019). The perturbation kinetic energy budget is first shown in table 3 . As for $T a_{m}=0$, the buoyancy contribution is really negligible, and the destabilization comes from the shear of the basic flow, with two main terms, $E_{s x}^{\prime}$ and $E_{s y}^{\prime}$. If the energy budget for $\operatorname{Pr}=0.00019$ (just above the transition at $\left.P r_{t}\right)$ is similar to what was found for $T a_{m}=0$ just above $P r_{t, 0}$, with a main destabilizing shear contribution coming from $E_{s y}^{\prime}$ and a smaller one coming from $E_{s x}^{\prime}$, changes appear for the two other values of $P r$. For these two cases, as already observed in $\S 5.2$ for $P r=0$ and non-zero $T a_{m}$, the dominant destabilizing shear term is $E_{s x}^{\prime}$ and $E_{s y}^{\prime}$ appears as stabilizing. Note that these terms become smaller (in absolute value) when $\mathrm{Pr}$ is increased. The analysis of the shear terms shows that for $\operatorname{Pr}=0.00018$, the structure of the global shear energy field $\mathscr{E}_{s}^{\prime}$, with three peaks connected to different peaks of $\mathscr{E}_{s x}^{\prime}$ and $\mathscr{E}_{s v}^{\prime}$, is really similar to what is shown for $P r=0$ and $T a_{m}=80$ in figure 15, and a similar analysis of the shear terms influenced by the RMF can be conducted. For $P r=0.00019$, the global shear energy $\mathscr{E}_{s}^{\prime}$ is close to what was found for $P r=0.00029$ at $T a_{m}=0$ (figure $11 a^{\prime}$ ) and the contributions $\mathscr{E}_{s x}^{\prime}$ and $\mathscr{E}_{s y}^{\prime}$ are as well, although some dissymmetries appear. The influence of the RMF, which corresponds to modifications of the shear energy field with the growth of a central peak, is then really effective only for very small values of the Prandtl number, below the transition at $\operatorname{Pr}_{t}$.

\section{Conclusion}

The effect of an RMF on the steady convective flow driven by a longitudinal temperature gradient in a horizontal channel of circular cross-section and on the stability of this flow has been studied.

Both theoretical and numerical approaches have been used to calculate the convective flows. In the case without an RMF (where the problem depends on the Grashof and Prandtl numbers), the fluid moves in the direction of the imposed temperature gradient in the lower part of the channel and in the opposite direction in the upper part. At non-zero Prandtl number, temperature gradients appear in the circular cross-section between the conducting boundaries and the central region of the channel. These temperature gradients are the result of the heat transported by the convective flow in the upper part and of the cooling induced by the flow in the lower part. They generate four vortices in the cross-section, which, for large enough values of the Prandtl and Grashof numbers, will lead to modifications of the longitudinal convective flow, as already observed in channels with rectangular cross-sections.

The effect of the RMF (parametrized by the magnetic Taylor number $T a_{m}$ and expressed by a purely azimuthal force proportional to the radial coordinate) is to induce a rotating motion of the fluid around the cylinder axis.

(i) For zero Prandtl number, a case where an analytical solution is derived, the flow in the circular cross-section corresponds to circular streamlines, and the longitudinal velocity structure is moved in the direction of the magnetic field rotation. The interface between the counterflows evolves from its horizontal position for $T a_{m}=0$ to a vertical orientation in the limit case of large $T a_{m}$ values. The intensity of the longitudinal 
flow, proportional to $G r$, decreases as $T a_{m}$ is increased and an asymptotic variation as $1 / T a_{m}$ (valid for $T a_{m}>200$ ) is obtained at large $T a_{m}$.

(ii) For non-zero Prandtl numbers but in the small $\mathrm{Pr}$ approximation (the temperature is not transported by the rotating flow), different limit cases are analysed. For small $T a_{m}$, the longitudinal velocity and the temperature field are not modified by the RMF, but the flow in the cross-section evolves from the four vortices structure to the circular streamlines according to the variations of the parameter $a=\left(11520 T a_{m}\right) /\left(\operatorname{Pr} G r^{2}\right)$. For large $T a_{m}$, a case where the longitudinal velocity field $W$ is transported by the rotation, the temperature field follows the transformations of $W$ and, as a consequence, the interface between hot and cold fluids also becomes vertical in the bulk in the asymptotic limit of large $T a_{m}$.

(iii) For finite Prandtl numbers, depending on the respective values of $\mathrm{Pr}, \mathrm{Gr}$, and $T a_{m}$, a great variety of flow structures can be obtained. These flow structures look like those revealed by the zero and small $\mathrm{Pr}$ approximations. They range from the structures with circular streamlines and transport by rotation of both longitudinal velocity and temperature field to the structures almost insensitive to rotation, with four cross-section vortices. As expected, it is found that the structures with circular streamlines are favoured by an increase of $T a_{m}$ or a decrease of $\mathrm{Pr}$ and $\mathrm{Gr}$. For nonzero $P r$, the intensity of the longitudinal flow $W$ also decreases as $T a_{m}$ is increased, but this decrease occurs later as $\operatorname{Pr}$ is increased and evolves more quickly. The curves $W\left(T a_{m}\right)$ eventually reach the asymptotic variation in $1 / T a_{m}$ found for $\operatorname{Pr}=0$. Finally, in the limit of large $T a_{m}$, it can be shown that the temperature field will be much affected by rotation and the interface between hot and cold fluids in the bulk will be horizontal, as the result of a $\pi$ rotation.

These convective flows in infinite cylindrical channels are invariant by translation $T_{z_{0}}$ along the cylinder axis. In the case without a magnetic field, they have two additional symmetries, a reflection $S_{l}$ with respect to the longitudinal vertical plane $V_{l}$ and a $\pi$ rotation $R_{\pi}$ about horizontal transverse axes as the $y$-axis (the combination of these two symmetries gives a symmetry $S_{c}$ with respect to points on the cylinder axis, as the point $C$ ). In the case with an RMF, the convective flows have only one additional symmetry, the symmetry $S_{c}$.

The linear stability analysis of these convective flows was first performed without a magnetic field in a range of small $\operatorname{Pr}$ values $\left(10^{-5} \leqslant P r \leqslant 0.02\right)$, and then with an RMF, at $P r=0$ for varying $T a_{m}$ and at fixed $T a_{m}$ values for varying $P r$. The results without a magnetic field show that in this $P r$ range the more dangerous modes are steady hydrodynamic modes. As $P r$ is increased, there is a steep increase of the thresholds at a given value $\operatorname{Pr}_{t, 0}$ of the Prandtl number. This corresponds to the stabilization (by a stable temperature stratification) of the usual shear mode related to the development of perturbations at the counter-stream boundary, and to the transition to a new shear mode, denoted as sidewall mode. This new mode develops closer to the sidewalls because the four cross-section rolls lead to an increase of the shear in these zones compared to the central part of the channel. The thresholds for this sidewall mode follow a characteristic law of decrease, $\mathrm{Gr}_{c} \sim \mathrm{Pr}^{-2 / 5}$, for $\operatorname{Pr}_{t, 0}<\operatorname{Pr}<0.02$. These stability results for $T a_{m}=0$ look similar to those obtained in the case of channels with a rectangular cross-section (transverse aspect ratio ranging from 1 to 5). The value of $\operatorname{Pr}_{t}$ is, however, still smaller in the cylindrical channel and the critical perturbations have broken the left-right $S_{l}$ symmetry. For the hydrodynamic instability at very small $\mathrm{Pr}$, the shear (mainly connected to the vertical shear $-\partial W_{0} / \partial y$ ) appears near the centre but, due to the symmetry breaking, the perturbation kinetic energy appears with two peaks, one on each side of the $V_{l}$ 


$\begin{array}{cccc} & P r=0 & P r=0.00018 & P r=0.00019 \\ T a_{m}=100 & G r_{c}=58357 & G r_{c}=20113 & G r_{c}=73664 \\ E_{b}^{\prime} & k_{c}=0.21 & k_{c}=0.32 & k_{c}=0.33 \\ E_{s}^{\prime} & 0 & -4 \times 10^{-5} & -6 \times 10^{-5} \\ E_{s x}^{\prime} & 1 & \approx 1 & \approx 1 \\ E_{s y}^{\prime} & 2.457 & 1.259 & 0.310 \\ & -1.457 & -0.260 & 0.688\end{array}$

TABLE 3. Kinetic energy budgets associated with the hydrodynamic modes at threshold for different values of the Prandtl number in the case with RMF $\left(T a_{m}=100\right)$. $P r=0.00018$ and 0.00019 are chosen below and above the transition at $\operatorname{Pr}_{t}$, respectively. Only the main shear contributions $E_{s x}^{\prime}$ and $E_{s y}^{\prime}$ are given. The other contributions exist but are small. (For $P r=0$ and 0.00018 , the largest one is connected to $\left(\partial U_{0} / \partial y\right)$ and its values are $4.0 \times 10^{-4}$ and $1.16 \times 10^{-3}$, respectively. For $\operatorname{Pr}=0.00019$, it is connected to $\left(\partial U_{0} / \partial x\right)$ and its value is $8.2 \times 10^{-4}$.)

plane. For the sidewall instability, two new peaks closer to the boundaries become dominant.

A very strong stabilization of the convective flows by the RMF is found for $\operatorname{Pr}=0$. The increase of the threshold can be fitted by an exponential law: $G r_{c} / G r_{c}\left(T a_{m}=\right.$ $0) \propto \exp \left(1.5 \times 10^{-5} \mathrm{Ta}_{m}^{2.6}\right)$. At the threshold, for $\operatorname{Pr}=0$, the RMF leads to the appearance at the centre of the channel of a new peak of perturbation kinetic energy connected now to the horizontal shear $-\partial W_{0} / \partial x$, and the two peaks connected to $-\partial W_{0} / \partial y$ are shifted closer to the boundaries and decrease in intensity. A similar type of stabilization, but less efficient, is obtained for small non-zero Prandtl numbers, until the transition Prandtl number $P r_{t}$ is reached. In fact, $P r_{t}$ is found to decrease when $T a_{m}$ is increased, but above $P r_{t}$, in spite of the RMF, a sidewall instability very similar to that described for $T a_{m}=0$ is obtained. In the $P r$ range corresponding to this sidewall instability at $T a_{m}=0$, the stabilization by the RMF is weak, almost negligible for $T a_{m}$ about a few hundred. This is due to the fact that in this $\operatorname{Pr}$ range, the thresholds $G r_{c}$ are high at $T a_{m}=0$, so that the basic flow at these values of $G r$ and $\operatorname{Pr}$ has very little sensitivity to the influence of $T a_{m}$.

The stabilization of the convective flows in a cylindrical channel by an RMF is then efficient for small values of $\operatorname{Pr}$ below $\operatorname{Pr} r_{t, 0}$, i.e. $\operatorname{Pr} \lesssim 3 \times 10^{-4}$, and particularly for very small values of $\operatorname{Pr}$ or for values of $\operatorname{Pr}$ close below $\operatorname{Pr}_{t, 0}$. For values of $\operatorname{Pr}$ above $\operatorname{Pr}_{t, 0}$, the instability thresholds (which correspond to the sidewall instability) are, without a magnetic field, stronger than those obtained for very small $\operatorname{Pr}$ (particularly for $\mathrm{Pr}$ values close above $P r_{t, 0}$ ), but the use of an RMF will not afford much stabilization in these cases, even for rather strong values of $T a_{m}$.

\section{REFERENCES}

Barz, R. U., Gerbeth, G., Wunderwald, U., Buhrig, E. \& Gelfgat, Y. M. 1997 Modelling of the isothermal melt flow due to rotating magnetic fields in crystal growth. J. Cryst. Growth 180, 410-421.

Bejan, A. \& Tien, C. L. 1978 Fully developed natural counterflow in a long horizontal pipe with different end temperatures. Intl J. Heat Mass Transfer 21, 701-708.

Ben Hadid, H., Vaux, S. \& KadDEChe, S. 2001 Three-dimensional flow transitions under a rotating magnetic field. J. Cryst. Growth 230, 57-62. 
Bontoux, P., Smutek, C., Roux, B. \& Lacroix, J. M. 1986 Three-dimensional buoyancy-driven flows in cylindrical cavities with differentially heated endwalls. Part 1 . Horizontal cylinders. J. Fluid Mech. 169, 211-227.

Braunsfurth, M. G. \& Mullin, T. 1996 An experimental study of oscillatory convection in liquid gallium. J. Fluid Mech. 327, 199-219.

Davoust, L., Cowley, M. D., Moreau, R. \& Bolcato, R. 1999 Buoyancy-driven convection with a uniform magnetic field. Part 2. Experimental investigation. J. Fluid Mech. 400, 59-90.

Dold, P. \& Benz, K. W. 1995 Convective temperature fluctuations in liquid gallium in dependence on static and rotating magnetic fields. Cryst. Res. Technol. 30, 1135-1145.

Dold, P. \& BenZ, K. W. 1997 Modification of fluid flow and heat transport in vertical Bridgman configurations by rotating magnetic fields. Cryst. Res. Technol. 32, 51-60.

Dold, P. \& Benz, K. W. 1999 Rotating magnetic field: fluid flow and crystal growth applications. Prog. Cryst. Growth Charact. Mater. 38, 7-38.

Gelfgat, A. Y., Bar-Yoseph, P. Z. \& Yarin, A. L. 1999 Stability of multiple steady states of convection in laterally heated cavities. J. Fluid Mech. 388, 315-334.

Grants, I. \& Gerbeth, G. 2001 Stability of axially symmetric flow driven by a rotating magnetic field in a cylindrical cavity. J. Fluid Mech. 431, 407-426.

Grants, I. \& Gerbeth, G. 2002 Linear three-dimensional instability of a magnetically driven rotating flow. J. Fluid Mech. 463, 229-239.

Hart, J. E. 1972 Stability of thin non-rotating Hadley circulations. J. Atmos. Sci. 29, 687-697.

HenRy, D. \& Ben Hadid, H. 2007 Multiple flow transitions in a box heated from the side in low-Prandtl-number fluids. Phys. Rev. E 76, 016314.

Henry, D. \& Buffat, M. 1998 Two- and three-dimensional numerical simulations of the transition to oscillatory convection in low-Prandtl-number fluids. J. Fluid Mech. 374, 145-171.

Hof, B., Juel, A., Zhao, L., Henry, D., Ben Hadid, H. \& Mullin, T. 2004 On the onset of oscillatory convection in molten gallium. J. Fluid Mech. 515, 391-413.

Hurle, D. T. J., Jakeman, E. \& Johnson, C. P. 1974 Convective temperature oscillations in molten gallium. J. Fluid Mech. 64 (3), 565-576.

Kaiser, T. \& Benz, K. W. 1998 Taylor vortex instabilities induced by a rotating magnetic field: a numerical approach. Phys. Fluids 10, 1104-1110.

Kamotani, Y. \& Sahraoui, T. 1990 Oscillatory natural convection in rectangular enclosures filled with mercury. J. Heat Transfer 112, 235-255.

Kuo, H. P. \& Korpela, S. A. 1988 Stability and finite amplitude natural convection in a shallow cavity with insulated top and bottom and heated from a side. Phys. Fluids 31, 33-42.

Laure, P. \& Roux, B. 1987 Synthèse des résultats obtenus par l'étude de stabilité des mouvements de convection dans une cavité horizontale de grande extension. C. R. Acad. Sci. Paris 305, $1137-1143$.

Lyubimov, D. V., Lyubimova, T. P. \& Morozov, V. A. 2001 Software package for numerical investigation of linear stability of multidimensional flows. Bull. Perm Univ.: Inf. Syst. Technol. 5, 74-81.

Lyubimova, T. P., Lyubimov, D. V., Morozov, V. A., Scuridin, R. V., Ben Hadid, H. \& Henry, D. 2009 Stability of convection in a horizontal channel subjected to a longitudinal temperature gradient. Part 1. Effect of aspect ratio and Prandtl number. J. Fluid Mech. 635, 275295.

Lyubimov, D. V., Lyubimova, T. P., Perminov, A. B., Henry, D. \& Ben Hadid, H. 2009 Stability of convection in a horizontal channel subjected to a longitudinal temperature gradient. Part 2. Effect of a magnetic field. J. Fluid Mech. 635, 297-319.

Martin Witkowski, L., Walker, J. S. \& Marty, P. 1999 Nonaxisymmetric flow in a finite-length cylinder with a rotating magnetic field. Phys. Fluids 11, 1821-1826.

MoreaU, R. 1990 Magnetohydrodynamics. Kluwer Academic.

Mössner, R. \& Gerbeth, G. 1999 Buoyant melt flows under the influence of steady and rotating magnetic fields. J. Cryst. Growth 197, 341-354.

Müller, A. \& Wiehelm, M. 1964 Periodische temperaturschwankungen in flüssigem InSb als ursache schichtweisen einbaus von Te in kristallisierendes InSb. Z. Naturforsch. A 19, 254 263.

Roux, B. (Ed.) 1990 Numerical simulation of oscillatory convection in low-Pr fluids. GAMM Workshop, Notes on Numerical Fluid Mechanics, vol. 27. Vieweg. 
Skeldon, A. C., Riley, D. C. \& Cliffe, K. A. 1996 Convection in a low-Prandtl-number fluid. J. Cryst. Growth 162, 95-106.

Utech, H. P. \& Flemings, M. C. 1966 Elimination of solute banding in Indium Antimonide crystals by growth in a magnetic field. J. Appl. Phys. 37 (5), 2021-2024.

Vaux, S., Ben Hadid, H. \& Henry, D. 2006 Study of the hydrodynamic instabilities in a differentially heated horizontal circular cylinder corresponding to a Bridgman growth configuration. J. Cryst. Growth 290, 674-682.

Volz, M. P. \& Mazuruk, K. 1999 Thermoconvective instability in a rotating magnetic field. Intl $J$. Heat Mass Transfer 42, 1037-1045.

Volz, M. P. \& Mazuruk, K. 2001 An experimental study of the influence of a rotating magnetic field on Rayleigh-Bénard convection. J. Fluid Mech. 444, 79-98.

WAKITANI, S. 2001 Numerical study of three-dimensional oscillatory natural convection at low Prandtl number in rectangular enclosures. J. Heat Transfer 123, 77-83.

Winters, K. H. 1988 Oscillatory convection in liquid metals in a horizontal temperature gradient. Intl J. Numer. Meth. Engng 25, 401-414. 